OPEN ACCESS

Edited by:

Chaowei Yang

George Mason University,

United States

Reviewed by:

Randall J. Olsen,

Houston Methodist Research Institute,

United States

Mohammad Saud Khan,

Brown University, United States

*Correspondence:

Rahul Kashyap

kashyap.rahu@mayo.edu

tThese authors have contributed equally to this work

Specialty section:

This article was submitted to

Intensive Care Medicine and Anesthesiology,

a section of the journal

Frontiers in Medicine

Received: 20 November 2020

Accepted: 17 February 2021

Published: 09 April 2021

Citation:

Bansal V, Mahapure KS, Mehra I, Bhurwal A, Tekin A, Singh R, Gupta I, Rathore SS, Khan H, Deshpande S,

Gulati S, Armaly P, Sheraton M and Kashyap R (2021) Mortality Benefit of Convalescent Plasma in COVID-19: A Systematic Review and Meta-Analysis. Front. Med. 8:624924. doi: 10.3389/fmed.2021.624924

\title{
Mortality Benefit of Convalescent Plasma in COVID-19: A Systematic Review and Meta-Analysis
}

\begin{abstract}
Vikas Bansal ${ }^{1 \dagger}$, Kiran S. Mahapure ${ }^{2 \dagger}$, Ishita Mehra ${ }^{3 \dagger}$, Abhishek Bhurwal ${ }^{4}$, Aysun Tekin ${ }^{1}$, Romil Singh ${ }^{5}$, Ishita Gupta ${ }^{6}$, Sawai Singh Rathore ${ }^{7}$, Hira Khan ${ }^{8}$, Sohiel Deshpande ${ }^{9}$, Shivam Gulati ${ }^{10}$, Paige Armaly ${ }^{11}$, Mack Sheraton ${ }^{12}$ and Rahul Kashyap ${ }^{1 *}$
\end{abstract}

\begin{abstract}
${ }^{1}$ Department of Anaesthesiology and Critical Care Medicine, Mayo Clinic, Rochester, MN, United States, ${ }^{2}$ Senior Resident, Department of Plastic Surgery, KAHER J. N. Medical College, Belgaum, India, ${ }^{3}$ Department of Internal Medicine, North Alabama Medical Center, Florence, AL, United States, ${ }^{4}$ Department of Gastroenterology and Hepatology, Rutgers Robert Wood Johnson School of Medicine, New Brunswick, NJ, United States, ${ }^{5}$ Department of Internal Medicine, Metropolitan Hospital, Jaipur, India, ${ }^{6}$ Department of Internal Medicine, Dr. Rajendra Prasad Government Medical College, Tanda, India, ${ }^{7}$ Department of Internal Medicine, Dr. Sampurnanand Medical College, Jodhpur, India, ${ }^{8}$ Department of Internal Medicine, Riphah International University Islamic International Medical College, Rawalpindi, Pakistan, ${ }^{9}$ Department of Internal Medicine, Maharashtra Institute of Medical Education and Research, Pune, India, ${ }^{10}$ Department of Internal Medicine, Adesh Institute of Medical Sciences and Research, Bathinda, India, "Department of Internal Medicine, University of the West Indies, Nassau, Bahamas, ${ }^{12}$ Department of Emergency Medicine, Trinity West Medical Center MSOPTI EM Program, Steubenville, OH, United States
\end{abstract}

Importance/Background: With a scarcity of high-grade evidence for COVID-19 treatment, researchers and health care providers across the world have resorted to classical and historical interventions. Immunotherapy with convalescent plasma (CPT) is one such therapeutic option.

Methods: A systematized search was conducted for articles published between December 2019 and 18th January 2021 focusing on convalescent plasma efficacy and safety in COVID-19. The primary outcomes were defined as mortality benefit in patients treated with convalescent plasma compared to standard therapy/placebo. The secondary outcome was pooled mortality rate and the adverse event rate in convalescent plasma-treated patients.

Results: A total of 27,706 patients were included in the qualitative analysis, and a total of 3,262 (2,127 in convalescent plasma-treated patients and 1,135 in the non-convalescent plasma/control group) patients died. The quantitative synthesis in 23 studies showed that the odds of mortality in patients who received plasma therapy were significantly lower than those in patients who did not receive plasma therapy [odds ratio (OR) 0.65 , $95 \%$ confidence interval $\left.(\mathrm{Cl}) 0.53-0.80, p<0.0001, l^{2}=15 \%\right)$. The mortality benefit remains the same even for 14 trials/prospective studies (OR 0.59, 95\% Cl 0.43-0.81, $p=0.001, l^{2}=22 \%$ ) as well as for nine case series/retrospective observational studies (OR $0.78,95 \% \mathrm{Cl} 0.65-0.94, p=0.01, l^{2}=0 \%$ ). However, in a subgroup analysis for 10 randomized controlled trials (RCTs), there was no statistically significant reduction in mortality between the CPT group compared to the non-CPT group (OR 0.76, 95\% Cl $\left.0.53-1.08, p=0.13, I^{2}=7 \%\right)$. Furthermore, the sensitivity analysis of $10 \mathrm{RCTs}$, excluding the study with the highest statistical weight, displayed a lower mortality rate compared 
to that of non-CPT COVID-19 patients (OR 0.64, 95\% Cl 0.42-0.97, $p=0.04, I^{2}=0 \%$ ). The observed pooled mortality rate was $12.9 \%$ (95\% Cl 9.7-16.9\%), and the pooled adverse event rate was $6.1 \%(95 \% \mathrm{Cl} 3.2-11.6)$, with significant heterogeneity.

Conclusions and Relevance: Our systemic review and meta-analysis suggests that CPT could be an effective therapeutic option with promising evidence on the safety and reduced mortality in concomitant treatment for COVID-19 along with antiviral/antimicrobial drugs, steroids, and other supportive care. Future exploratory studies could benefit from more standardized reporting, especially in terms of the timing of interventions and clinically relevant outcomes, like days until discharge from the hospital and improvement of clinical symptoms.

Keywords: COVID-19, SARS-CoV 2, mortality, plasma therapy, systemic review and meta-analysis, convalescent plasma

\section{HIGHLIGHTS}

\section{What we Already Know About This Topic}

- COVID-19 is an ongoing global pandemic, for which convalescent plasma has been recommended as a possible therapeutic drug.

- Preliminary clinical trial results propose that there may be a satisfactory safety profile and better clinical outcome for patients treated with convalescent plasma compared with those treated with placebo or were under standard of care; however, data are limited at the current time.

\section{What This Article Tells Us That Is New}

- This systematic review and meta-analysis provides an exhaustive summary of current literature on the efficacy and safety of convalescent plasma use in COVID-19 patients.

\section{INTRODUCTION}

The first case of coronavirus was identified in Wuhan, China, at the end of 2019 (1). The World Health Organization (WHO) declared a public health emergency of international concern on 30th January 2020 and a global pandemic on 11th March 2020 (2). The WHO estimates that serious illness occurs in $13.8 \%$ of cases and that $6.1 \%$ cases are critical (3). As of 3rd February 2021, there have been 104,077,986 confirmed cases of COVID-19, including 2,259,391 deaths, reported worldwide (4).

Severe Acute Respiratory Syndrome Coronavirus 2 (SARS$\mathrm{CoV}-2$ ) is an RNA virus that is believed to primarily affect the respiratory tract; however, numerous complications related to systems other than the respiratory system have also been noted (5). Even though certain drugs, such as remdesivir, have been repositioned for emergency use in COVID-19, no particular drugs have yet been identified as an effective treatment of COVID-19. Therefore, various clinical trials are ongoing in

Abbreviations: COVID-19, Coronavirus Disease 2019; CPT, immunotherapy with convalescent plasma; FDA, Food and Drug Administration; HIV, human immunodeficiency virus; RNA, ribonucleotide acid; SARS-CoV-2, Severe Acute Respiratory Syndrome Coronavirus 2; TRALI, transfusion-related acute lung injury; WHO, World Health Organization. search for the best therapy. With a scarcity of high-grade evidence for COVID-19 treatment, researchers and health care providers across the world have resorted to classical and historical interventions. Immunotherapy with convalescent plasma (CPT) is one such therapeutic option.

Convalescent plasma uses have been well-described in various diseases such as severe acute respiratory syndrome (6), Middle East respiratory syndrome coronavirus (6), Ebola virus disease (7), pandemic influenza A (6), and avian-origin influenza A (6), and a neutralizing antibody response directed against the viral $\mathrm{S}$ protein of the SARS virus has been reported (8). The antibodies primarily target the trimeric spike (S) surface glycoproteins, which are used by the virus to enter the host cells (9). The antibody thus hinders the ability of the SARS-CoV-ACE2 to enter the host cells and can be detected even 24 months after the onset of infection (9). Subsequently, the Food and Drug Administration (FDA) approved the use of convalescent plasmas for patients with serious or immediately life-threatening COVID19 infections on 24th March 2020 (10).

One of the first studies demonstrating the benefit of CPT was reported in April 2020 (11). Since then, there has been increasing interest $(12,13)$, and three inconclusive Cochrane reviews (14-16) revealed that unmatched cohort studies are still the most frequent reports. As the literature around CPT is evolving and newer studies are being reported across the world, we conducted a systematic review and meta-analysis to appraise the currently available data for the clinical usefulness of convalescent plasma for the treatment of COVID-19. Organizing summaries of the available clinical evidence regarding safety and effectiveness from published literature through a systematic review can provide a synopsis of clinical evidence on the potential benefits and adverse events of CPT therapy in critically ill COVID-19 patients.

\section{METHODS}

Our study has been performed in accordance with the Preferred Reporting Items for Systematic Reviews and Meta-analyses (PRISMA) statement $(17,18)$. 


\section{Search Strategy}

The search strategy was designed and conducted by the authors (IM, KM, and VB). A systematic search was conducted from COVID-19 inception through 7th August 2020 for full-length articles focusing on the efficacy and safety of convalescent plasma in COVID-19 in three major COVID-19 research article databases, namely, WHO Global Research Database, CDC COVID-19 Research Articles Downloadable Database, and LitCovid database. These databases automatically gathered for articles related to COVID-19. Other literature sources such as the Eurosurveillance, China CDC Weekly, Homeland Security Digital Library, ClinicalTrials.gov, bioRxiv (preprints), medRxiv (preprints), chemRxiv (preprints), and SSRN (preprints) were searched as well. The search strategy consisted of a combination of keywords such as "Convalescent Plasma, Plasma therapy, COVID-19, SARS-CoV 2, Mortality, Systemic, Review, Metaanalysis" across the combined COVID-19 databases. After a thorough search was performed, full-length articles meeting the inclusion criteria were evaluated. All titles and abstracts were identified by the authors and screened to accrue potentially eligible studies. A manual search of the references of the included studies was also performed to supplement the electronic search. Then, the same reviewers (AT, IG, KM, PA, RS, and SG) independently assessed all selected full-text manuscripts for eligibility.

\section{Eligibility Criteria}

The specific inclusion criteria for the systematic review and metaanalysis were as follows: (1) all RCTs or prospective studies or retrospective studies in hospitalized patients with COVID19, (2) the use of plasma as therapy for COVID-19, (3) all studies with information available to evaluate the incidence of mortality in COVID-19 patients with plasma use [number of events, sample size, odds ratio (OR), and confidence interval (CI)], and (4) full-text articles. Thus, reviewed studies included in our analysis were RCTs and prospective and retrospective studies evaluating the outcomes of plasma therapy in COVID-19 patients. Studies focusing on patients $<18$ years of age, focusing on pregnant females, and limited to particular comorbidities and organ dysfunctions were excluded to avoid selection bias. We also excluded case reports from our systematic review.

\section{Data Extraction}

Once the studies met the inclusion criteria, four reviewers (AT, HK, IG, IM, KM, RS, or SD) independently reviewed and abstracted data for mortality rate and adverse event rate for each eligible study (Figure 1). If there were multiple reports stemming from a specific study database, data from the most robust study were extracted, with other studies contributing toward the bibliography. Subsequently, the data were collected and tabulated using Microsoft Excel. The included data were checked for accuracy by AB, KM, IG, and VB. The reviewers sorted the data separately in all stages of study collection, data extraction, and quality assessment. All discrepancies found between two reviewers were resolved with consensus and inputs from other authors.

\section{Study Characteristics and Quality Assessment}

Randomized trial and prospective studies were evaluated using the Cochrane risk-of-bias tool (19), and the correlation of quality measures with estimates of treatment effects in the meta-analyses of RCTs (20) was used for quality assessment of the same. We used the NIH Quality Assessment Tool for Case Series Studies (21) and the Newcastle-Ottawa Scale (NOS) (22) for casecontrol or non-randomized retrospective cohort studies. For each non-randomized study, we assessed the study design and content. The studies were then graded using a "star system" on the basis of (1) the selection of the study groups, (2) the comparability of the groups, and (3) the ascertainment of the outcome of interest. Quality assessments were also conducted independently, and discrepancies were resolved by consensus.

\section{Outcome Measures}

All the studies describing the outcomes of plasma therapy in patients with COVID-19 were analyzed in detail. Primary outcomes were mortality benefits for patients on CPT in COVID19. The mortality rate was evaluated in comparison to that of the control group (placebo or non-CPT). The defined secondary outcome was the pooled mortality rate and pooled adverse event rate.

\section{Quantitative Data Synthesis}

Primary outcomes were analyzed by the Review Manager (RevMan) computer program, version 5.4 for Windows (23), and the Comprehensive Meta-Analysis software package (BioStat, Englewood, NJ, USA) (24) was used for calculating the mortality and adverse event rates. The final pooled risk estimates were obtained using random effects models (25). Raw data for outcomes and non-events from each study were used to calculate crude OR with respective $95 \%$ CI for each study. The Cochrane $Q$ and the $I^{2}$ statistics were calculated to assess heterogeneity between studies $(25,26) . p<0.10$ for chi-square tests and $I^{2}<$ $20 \%$ were interpreted as low-level heterogeneity. We planned to perform a subgroup analysis by study design (trial/prospective studies and observational) to decrease burden of selection bias of the observational studies. It is expected that the estimates from observational studies will be more overestimated than those from RCTs (26). Furthermore, we planned to conduct a sensitivity analysis for randomized trials in trial/prospective studies to check for robustness of the results. The probability of publication bias was assessed using funnel plots and Egger's tests.

\section{RESULTS}

The initial library search identified potentially relevant citations from the WHO Global Research Database, CDC COVID19 Research Articles Downloadable Database, and LitCovid PubMed database comprising 174,398 articles. Subsequently, 61,487 duplicates were removed. Out of the remaining 112,911 articles, a total of 2,262 focused on convalescent plasma. A total of 2,014 articles were excluded after title and abstract reviews due to not having patient data. We added 404 articles during literature update on 18th January 2021 in the initial literature 


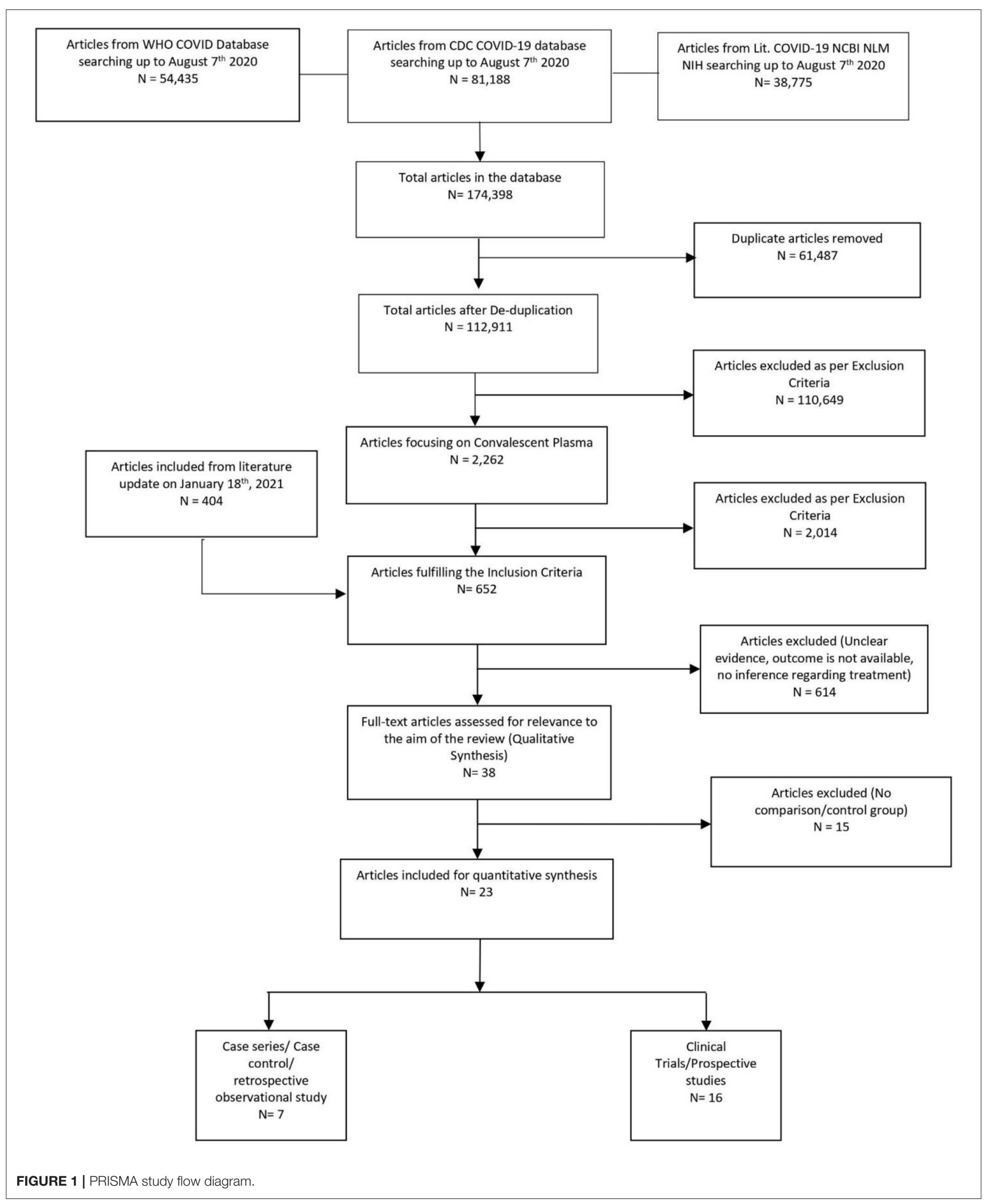


TABLE 1A | Study characteristics.

\begin{tabular}{|c|c|c|c|c|c|c|c|}
\hline References & Type of study & $\begin{array}{l}\text { Dose of } \\
\text { convalescent } \\
\text { plasma }\end{array}$ & $\begin{array}{l}\text { Hospital } \\
\text { length of } \\
\text { stay }\end{array}$ & $\begin{array}{l}\text { Antibodies } \\
\text { titer }\end{array}$ & $\begin{array}{l}\text { Viral } \\
\text { shedding }\end{array}$ & $\begin{array}{l}\text { Concomitant treatment } \\
\text { with CPT }\end{array}$ & Adverse events \\
\hline Agarwal et al. (28) & Trial & $200 \mathrm{ml}$ & 14 days & $\mathrm{N} / \mathrm{D}$ & $\mathrm{N} / \mathrm{D}$ & $\begin{array}{l}\text { Methylprednisolone, } \\
\text { prednisone, azithromycin, } \\
\text { hydroxychloroquine, lopinavir, } \\
\text { and ritonavir }\end{array}$ & $\begin{array}{l}\text { Pain in the local infusion site, } \\
\text { chills, nausea, bradycardia, } \\
\text { and dizziness reported in one } \\
\text { patient each. Fever and } \\
\text { tachycardia reported in three } \\
\text { patients each. Dyspnea and } \\
\text { intravenous catheter blockage } \\
\text { noted in two patients each. } \\
\text { Mortality assessed as possibly } \\
\text { related to convalescent } \\
\text { plasma (CP) transfusion in } \\
\text { three patients }\end{array}$ \\
\hline Altuntas et al. (54) & Case-control & 200-600 ml & $17-18$ days & $N / D$ & $\mathrm{~N} / \mathrm{D}$ & Antiviral azithromycin & N/A \\
\hline $\begin{array}{l}\text { Avenado-Sola et al. } \\
\text { (62) }\end{array}$ & $\begin{array}{l}\text { Multicenter } \\
\text { randomized } \\
\text { clinical trial }\end{array}$ & 250-300 ml & $\mathrm{N} / \mathrm{A}$ & $\mathrm{N} / \mathrm{A}$ & $\mathrm{N} / \mathrm{A}$ & Yes & $\begin{array}{l}\text { Six in } \mathrm{CP} \text {, seven in standard of } \\
\text { care (SOC) }\end{array}$ \\
\hline Bajpai et al. (59) & $\begin{array}{l}\text { Open-labeled } \\
\text { randomized } \\
\text { controlled trial } \\
(\mathrm{RCT})\end{array}$ & $500 \mathrm{ml}$ & 14 days & $>80$ & $\mathrm{~N} / \mathrm{A}$ & $\begin{array}{l}\text { Hydroxychloroquine, } \\
\text { azithromycin, and oseltamivir }\end{array}$ & $\begin{array}{l}\text { Mild urticaria in one patient } \\
\text { each of CP and fresh frozen } \\
\text { plasma (FFP) arms }\end{array}$ \\
\hline Donato et al. (30) & $\begin{array}{l}\text { Trial with matched } \\
\text { cohort study }\end{array}$ & $200-500 \mathrm{ml}$ & $\mathrm{N} / \mathrm{D}$ & $\begin{array}{l}1: 1,000- \\
10,000 \text { to } \\
>1: 10,000 \\
\text { in some } \\
\text { patients }\end{array}$ & $\mathrm{N} / \mathrm{D}$ & $\begin{array}{l}\text { Hydroxychloroquine, steroids, } \\
\text { remdesivir, azithromycin, and } \\
\text { tocilizumab }\end{array}$ & Mild rash in one patient \\
\hline Liu et al. (35) & Case-control & $250 \mathrm{ml}$ & $\mathrm{N} / \mathrm{D}$ & $\geq 1: 320$ & $\mathrm{~N} / \mathrm{D}$ & $\begin{array}{l}\text { Azithromycin, broad-spectrum } \\
\text { antibiotics, } \\
\text { hydroxychloroquine, antivirals, } \\
\text { corticosteroids, interleukin-6 } \\
\text { inhibitors, and therapeutic } \\
\text { anticoagulation }\end{array}$ & $\begin{array}{l}\text { No adverse reactions were } \\
\text { observed }\end{array}$ \\
\hline Hartman et al. (32) & Single-arm trial & $\mathrm{N} / \mathrm{D}$ & 12 days & $\mathrm{N} / \mathrm{D}$ & $\mathrm{N} / \mathrm{D}$ & Data unavailable & N/D \\
\hline Hegerova et al. (33) & Case-control & $\mathrm{N} / \mathrm{D}$ & 15 days & $\mathrm{N} / \mathrm{D}$ & $\mathrm{N} / \mathrm{D}$ & $\begin{array}{l}\text { Azithromycin and } \\
\text { hydroxychloroquine }\end{array}$ & $\begin{array}{l}\text { No adverse reactions were } \\
\text { observed }\end{array}$ \\
\hline Joyner et al. (12) & Clinical trial & $200-500 \mathrm{ml}$ & N/D & $\mathrm{N} / \mathrm{D}$ & $\mathrm{N} / \mathrm{D}$ & $\mathrm{N} / \mathrm{D}$ & $\begin{array}{l}\text { Transfusion reactions ( } n=78 \text {; } \\
<1 \% \text { ), thromboembolic or } \\
\text { thrombotic events ( } n=113 \text {; } \\
<1 \% \text { ), and cardiac events ( } \\
=677 \text { ) }\end{array}$ \\
\hline $\begin{array}{l}\text { Karekadavath et al. } \\
\text { (51) }\end{array}$ & Case series & $200 \mathrm{ml}$ & 22-43 days & $\mathrm{N} / \mathrm{D}$ & $\begin{array}{l}20-42 \\
\text { days }\end{array}$ & Remdesivir and ribavirin & $\mathrm{N} / \mathrm{D}$ \\
\hline Li et al. (34) & Trial & $\begin{array}{l}4-13 \mathrm{ml} / \mathrm{kg} \text { of } \\
\text { recipient body } \\
\text { weight }\end{array}$ & $7-28$ days & $\mathrm{N} / \mathrm{D}$ & $\mathrm{N} / \mathrm{D}$ & $\begin{array}{l}\text { Antiviral, interferon, Chinese } \\
\text { herbal medicine, antibacterial, } \\
\text { antifungal, steroids, and } \\
\text { human immunoglobulin }\end{array}$ & Seen in two patients \\
\hline Libster et al. (58) & $\begin{array}{l}\text { Double-blind } \\
\text { placebo RCT }\end{array}$ & $250 \mathrm{ml}$ & $\mathrm{N} / \mathrm{A}$ & $>1: 1,000$ & $\mathrm{~N} / \mathrm{A}$ & $\mathrm{N} / \mathrm{A}$ & $\mathrm{N} / \mathrm{A}$ \\
\hline
\end{tabular}


TABLE 1A | Continued

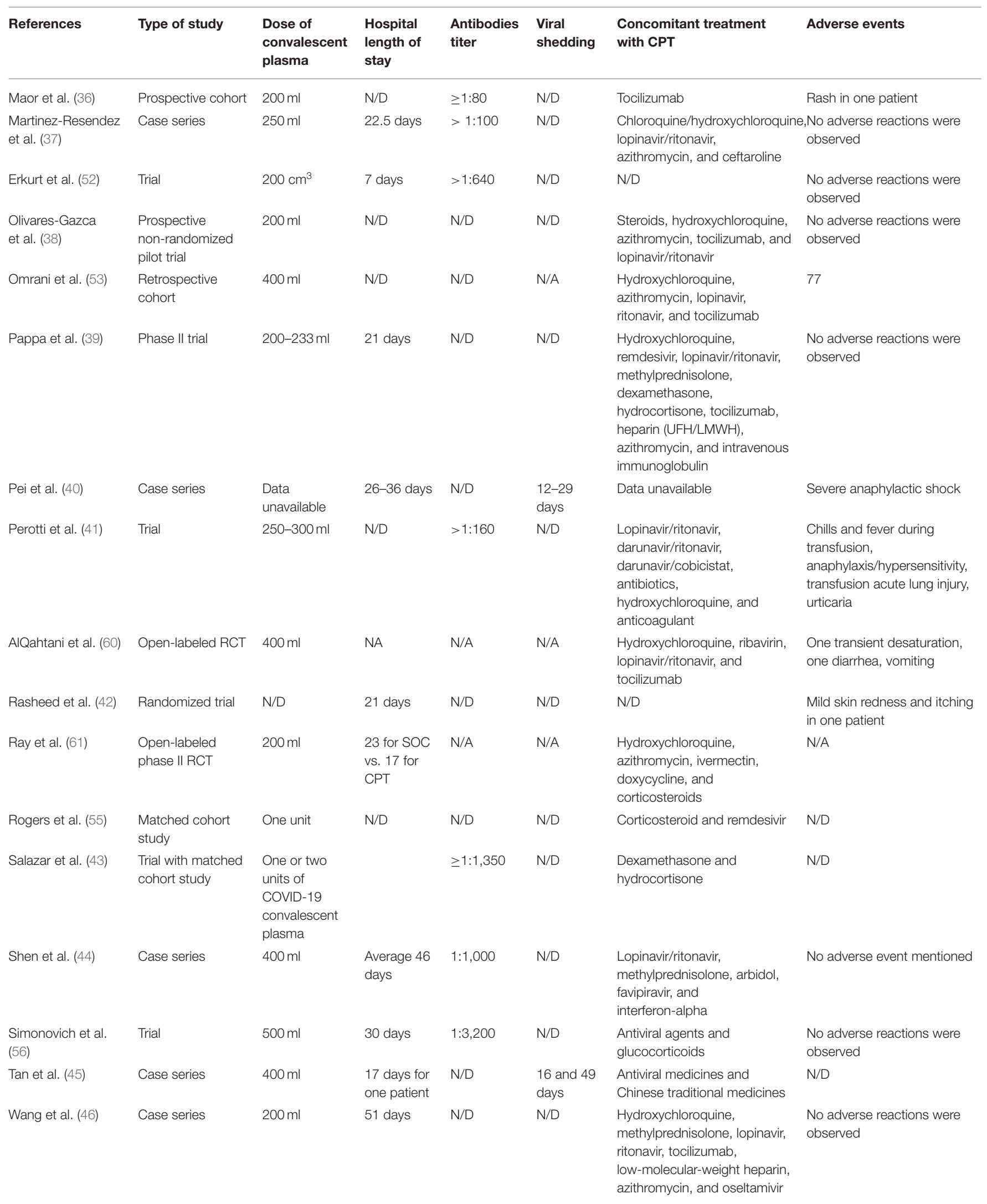


TABLE 1A | Continued

\begin{tabular}{|c|c|c|c|c|c|c|c|}
\hline References & Type of study & $\begin{array}{l}\text { Dose of } \\
\text { convalescent } \\
\text { plasma }\end{array}$ & $\begin{array}{l}\text { Hospital } \\
\text { length of } \\
\text { stay }\end{array}$ & $\begin{array}{l}\text { Antibodies } \\
\text { titer }\end{array}$ & $\begin{array}{l}\text { Viral } \\
\text { shedding }\end{array}$ & $\begin{array}{l}\text { Concomitant treatment } \\
\text { with CPT }\end{array}$ & Adverse events \\
\hline Xia et al. (47) & Case-control & $200-1,200 \mathrm{ml}$ & 22 days & $\mathrm{N} / \mathrm{D}$ & $\mathrm{N} / \mathrm{D}$ & N/D & $\begin{array}{l}\text { Minor allergic reactions } \\
\text { (pruritus or erythema) in three } \\
\text { patients }\end{array}$ \\
\hline Ye et al. (48) & Case series & $200 \mathrm{ml}$ & 15-24 days & $\mathrm{N} / \mathrm{D}$ & N/D & Arbidol and levofloxacin & $\begin{array}{l}\text { No adverse reactions were } \\
\text { observed }\end{array}$ \\
\hline Yoon et al. (57) & $\begin{array}{l}\text { Retrospective } \\
\text { cohort }\end{array}$ & $200 \mathrm{ml}$ & $\mathrm{N} / \mathrm{A}$ & $>1: 1,000$ & $\mathrm{~N} / \mathrm{A}$ & Corticosteroids & N/A \\
\hline Zeng et al. (49) & Case-control & $\begin{array}{l}300(200-600) \\
\mathrm{ml}\end{array}$ & N/D & $\mathrm{N} / \mathrm{D}$ & 23.5 days & $\begin{array}{l}\text { Glucocorticoid and traditional } \\
\text { Chinese medicine }\end{array}$ & $\begin{array}{l}\text { No adverse reactions were } \\
\text { observed }\end{array}$ \\
\hline Zhang et al. (50) & Case series & $200-2,400 \mathrm{ml}$ & $21-41$ days & $N / D$ & N/D & $\begin{array}{l}\text { Lopinavir/ritonavir, } \\
\text { methylprednisolone, arbidol, } \\
\text { favipiravir, interferon-alpha, } \\
\text { and oseltamivir }\end{array}$ & $\begin{array}{l}\text { No adverse reactions were } \\
\text { observed }\end{array}$ \\
\hline
\end{tabular}

N/A, not available; N/D, not defined/not mentioned.

search. The remaining 652 manuscripts were scrutinized further, and 615 were further excluded because of unclear evidence and non-relevance to the objective of the manuscript. Thus, 38 studies $(11,12,27-62)$ were included in their entirety, as shown in Table 1. The PRISMA flowchart is shown in Figure 1.

\section{Study Characteristics}

A total of 38 studies $(11,12,27-62)$ were included in the qualitative analysis (Tables 1A,B). Out of which, 23 studies $(11,27,28,30,31,33-35,41-43,47,49,53-62)$ compared the mortality in convalescent plasma-treated patients vs. that in patients treated by standard therapy/placebo. Out of 14 trials/prospective studies, 10 trials $(28,31,34,42,56,58-$ 62) conducted proper randomization, and six trials/prospective studies matched with the cohort retrospectively $(27,30,41,43)$. Zhang et al. (50) concluded that seroconversion occurred in 5-24 days, while Zeng et al. (49) mentioned that all six patients tested negative within 3 days of starting convalescent plasma. Tan et al. (45) evaluated the viral shedding period in convalescent plasmatreated patients, which was 16-46 days. Joyner et al. conducted the largest interventional case study with 20,000 convalescent plasma-treated patients and evaluated the safety profile (12).

\section{Primary Outcome Mortality Comparison Between Plasma Therapy and Placebo}

Twenty-three studies reported the mortality rate in COVID-19 patients on plasma and non-CPT therapy $(11,27,28,30,31,33-$ $35,41-43,47,49,53-62)$. This yielded a sample size of 7,542 patients, with 2,392 patients on plasma therapy and 5,150 patients in the control group. In the CPT therapy cohort, 392 patients died, while 1,135 patients died in the placebo/non-CPT cohort. The meta-analysis of these mortality rates showed that the odds of mortality on plasma therapy were significantly lower than those in patients who did not receive plasma therapy (OR 0.65 , 95\% CI $\left.0.53-0.80, p<0.0001, I^{2}=15 \%\right)$. This is shown in a Forrest plot (Figure 2A). We performed a subgroup analysis by study designs and observed similar mortality benefits in 14 trial/prospective studies $(27,28,30,31,34,41-43,56,58-62)$ (OR 0.59, 95\% CI 0.43-0.81, $p=0.001, I^{2}=22 \%$ ) (Figure 2B) as well as for nine case series/retrospective observational studies $(11,33,35,47,49,53-55,57)$ (OR 0.78, 95\% CI 0.65-0.94, p $=0.01, I^{2}=0 \%$ ) (Figure $2 \mathrm{C}$ ). However, during the sensitivity analysis of 10 randomized trials $(28,31,34,42,56,58-62)$, no statistically significant reduction of COVID-19 deaths was shown (OR 0.76, 95\% CI 0.53-1.08, $p=0.13, I^{2}=7 \%$ ) (Figure 2D). Agarwal et al. (28) demonstrated a different effect and had a large statistical weight $(34.3 \%)$. Therefore, a sensitivity analysis was performed by excluding the study by Agarwal et al. (28). This revealed a significant reduction in the odds of mortality with COVID-19 (OR 0.64, 95\% CI 0.42-0.97, $p=0.04, I^{2}=0 \%$ ) (Figure 2E).

\section{Secondary Outcome Pooled Mortality Rate}

Thirty-eight studies $(11,12,27-62)$ reported the mortality rate in COVID-19 patients on plasma therapy, as shown in Figure 3. A total of 22,556 patients with CPT were included in the analysis, of which a total of 2,127 patients died. This yielded a pooled post CPT mortality rate of $12.9 \%(95 \%$ CI 9.7-16.9) with a substantial amount of heterogeneity $\left(I^{2}=89.6\right)$ in the analysis (Figure 3 ).

\section{Pooled Adverse Event Rate}

Similarly, 37 studies $(11,12,27-53,55-62)$ reported the adverse event rate in COVID-19 patients on plasma therapy, as shown in Figure 4. A total of 21,668 patients with CPT were included in the analysis, of which a total of 1,506 patients had adverse events. This yielded a pooled adverse event rate of $6.1 \%$ (95\% CI 3.211.6) with significant heterogeneity in the analysis $\left(I^{2}=94.9\right)$ (Figure 4).

\section{Risk-of-Bias Assessment}

Two authors (KM and AT) independently assessed the risk of bias of each study included. All disagreements were discussed with 
A

Study or Subgroup Events Total Events Total Weight M-H, Random, $95 \% \mathrm{Cl}$

Abolgashemi et al

Agawal et al

Altuntas et al

Awendano-Sola et al

Bajpai et al

Donato et al

Duan et al

Gharbaran et al

H.liu et al

Hegerova et al

Libster et al

Ling Li et al

Omrani et al

Perotti et al

Qahtani et al

Rasheed et al

Ray et al

Rogers et al

Salazar et al

Simonovich et al

Xinyl xia et al

Yoon et al

Zeng et al

Total $(95 \% \mathrm{Cl})$

Total events

Heterogeneity: $\mathrm{Tau}^{\mathrm{z}}=0.03 ; \mathrm{Chi}^{\mathrm{z}}=25.89, \mathrm{df}=22(\mathrm{P}=0.26) ; \mathrm{I}^{\mathrm{z}}=15 \%$

Test for overall effect: $Z=4.09(\mathrm{P}<0.0001)$

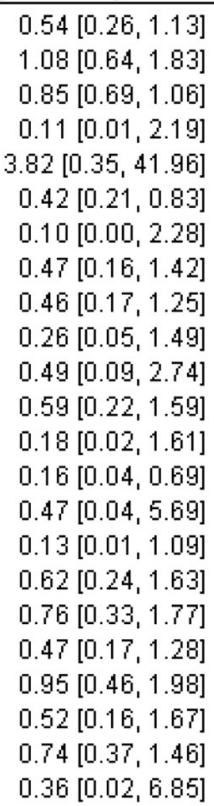

$0.36[0.02,6.85]$

$0.65[0.53,0.80]$
Odds Ratio

M-H, Random, $95 \% \mathrm{Cl}$

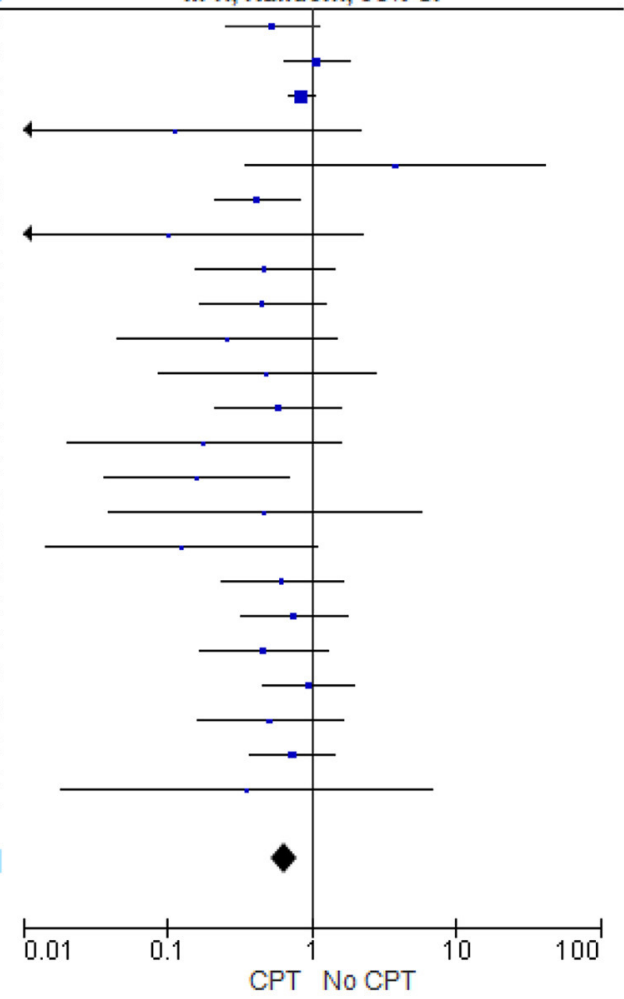

B

CPT No CPT Odds Ratio

Study or Subgroup Events Total Events Total Weight M-H, Random, 95\% Cl

Abolgashemi et al $\quad \begin{array}{llllll}17 & 115 & 18 & 74 & 121 \% & 0.54[0.26,1.13]\end{array}$

$\begin{array}{llllll}\text { Agawwal et al } & 34 & 235 & 31 & 229 & 18.1 \%\end{array}$

Avendano-Sola et al

$4-43-1.1 \%$

Bajpai et al

Donato et al

Gharbaran et al

$1 \quad 15 \quad 1.7 \%$

Libster et al

Ling Li et al

Perotti et al

Qahtani et al

Rasheed et al

Ray et al

Salazar et al

Simonovich et al

Total $(95 \% \mathrm{Cl})$

$0 \quad 38$

$\begin{array}{rrrrr}11 & 47 & 565 & 1340 & 13.4 \% \\ 6 & 43 & 11 & 43 & 6.7 \%\end{array}$

$\begin{array}{rrrrr}6 & 43 & 11 & 43 & 6.7 \% \\ 2 & 80 & 4 & 80 & 3.1 \%\end{array}$

$\begin{array}{lllll}8 & 51 & 12 & 50 & 7.9 \%\end{array}$

$\begin{array}{lllll}3 & 46 & 7 & 23 & 4.1 \%\end{array}$

$\begin{array}{lcr}\text { Total events } & 126 & 708\end{array}$

120

$220 \quad 1.6 \%$

$\begin{array}{lllll}1 & 21 & 8 & 28 & 2.0 \%\end{array}$

$\begin{array}{lllll}10 & 40 & 14 & 40 & 8.3 \%\end{array}$

$\begin{array}{lllll}5 & 136 & 19 & 251 & 7.7 \%\end{array}$

$1.08[0.64,1.83]$

$0.11[0.01,2.19]$

$3.82[0.35,41.96]$

$0.42[0.21,0.83]$

$0.47[0.16,1.42]$

$0.49[0.09,2.74]$

$0.59[0.22,1.59]$

$0.16[0.04,0.69]$

$0.47[0.04,5.69]$

$0.13[0.01,1.09]$

$0.62[0.24,1.63]$

$0.47[0.17,1.28]$

$0.95[0.46,1.98]$

$2341100.0 \%$

$0.59[0.43,0.81]$

Test for overall effect: $Z=3.26(\mathrm{P}=0.001)$

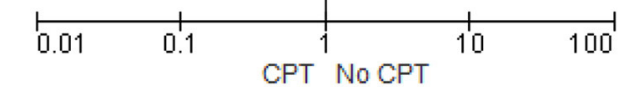

FIGURE 2 | 
C

CPT No CPT Odds Ratio

Study or Subgroup Events Total Events Total Weight M-H, Random, 95\% Cl Altuntas et al

Duan et al

H.liu et al

Hegerova et al

Omrani et al

Rogers et al

xinyl xia et al

Yoon et al

Zeng et al

Total $(95 \% \mathrm{Cl})$

Total events

Heterogeneity: $\mathrm{Tau}^{2}=0.00 ; \mathrm{Chi}^{2}=7.49, \mathrm{df}=8(\mathrm{P}=0.48) ; \mathrm{I}^{2}=0 \%$

Test for overall effect: $Z=2.56(P=0.01)$

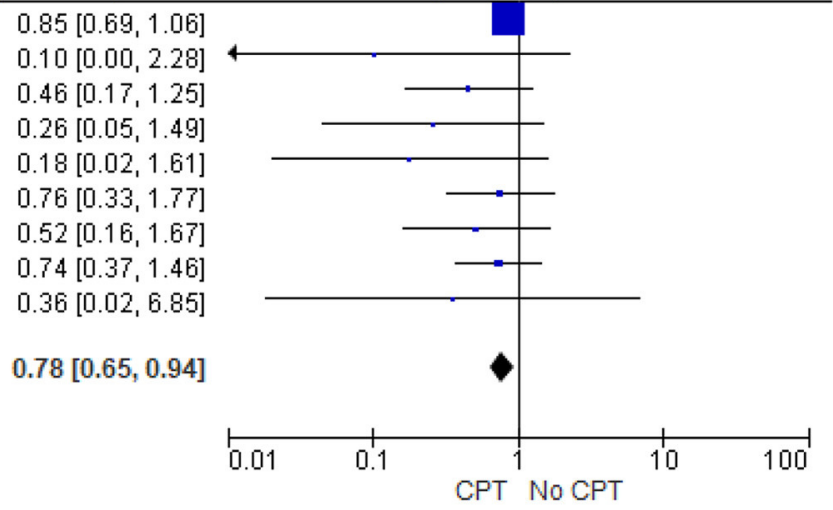

Odds Ratio

D

CPT No CPT

\begin{tabular}{lrrrrrr} 
Study or Subgroup & Events & Total & Events & Total & Weight & M-H, Random, 95\% Cl \\
\hline Agarwal et al & 34 & 235 & 31 & 229 & $34.3 \%$ & $1.08[0.64,1.83]$
\end{tabular}

$\begin{array}{llllll}\text { Agawal et al } & 34 & 235 & 31 & 229 & 34.3 \%\end{array}$

Awendano-Sola et al $\quad \begin{array}{rrrrr}0 & 38 & 4 & 43 & 1.4 \%\end{array}$

$\begin{array}{llllll}\text { Bajpai et al } & 3 & 14 & 1 & 15 & 2.1 \%\end{array}$

$\begin{array}{llllll}\text { Gharbaran et al } & 6 & 43 & 11 & 43 & 9.6 \%\end{array}$

$\begin{array}{llllll}\text { Libster et al } & 2 & 80 & 4 & 80 & 4.1 \%\end{array}$

Ling Li et al $\quad \begin{array}{llllll}8 & 51 & 12 & 50 & 11.6 \%\end{array}$

$\begin{array}{llllll}\text { Qahtani et al } & 1 & 20 & 2 & 20 & 2.0 \%\end{array}$

Rasheed et al

Ray et al

Simonovich et al

121

$828 \quad 2.6 \%$

$14 \quad 40 \quad 12.2 \%$

$0.11[0.01,2.19]$

$3.82[0.35,41.96]$

$0.47[0.16,1.42]$

$0.49[0.09,2.74]$

$0.59[0.22,1.59]$

$0.47[0.04,5.69]$

$0.13[0.01,1.09]$

$0.62[0.24,1.63]$

$0.95[0.46,1.98]$

Total $(95 \% \mathrm{Cl})$

770

$12 \quad 105 \quad 20.1 \%$

$0.76[0.53,1.08]$

$653100.0 \%$

$\begin{array}{lcc}\text { Total events } & 90 & 99 \\ \text { Heterogeneity: } \operatorname{Tau}^{2}=0.02 ; \mathrm{Chi}^{2}=9.65, \mathrm{df}=9(\mathrm{P}=0.38) ; \mathrm{I}^{2}=7 \%\end{array}$

Test for overall effect: $Z=1.53(\mathrm{P}=0.13)$

E

CPT No CPT

Odds Ratio

Study or Subgroup Events Total Events Total Weight M-H, Random, 95\% Cl $\begin{array}{llllll}\text { Agarwal et al } & 34 & 235 & 31 & 229 & 0.0 \%\end{array}$

Awendano-Sola et al $\quad \begin{array}{rrrrr}0 & 38 & 4 & 43 & 2.0 \%\end{array}$

$\begin{array}{llllll}\text { Bajpai et al } & 3 & 14 & 1 & 15 & 3.0 \%\end{array}$

$\begin{array}{llllll}\text { Gharbaran et al } & 6 & 43 & 11 & 43 & 14.3 \%\end{array}$

$\begin{array}{llllll}\text { Libster et al } & 2 & 80 & 4 & 80 & 5.8 \%\end{array}$

$\begin{array}{llllll}\text { Ling Li et al } & 8 & 51 & 12 & 50 & 17.5 \%\end{array}$

$\begin{array}{llllll}\text { Qahtani et al } & 1 & 20 & 2 & 20 & 2.8 \%\end{array}$

Rasheed et al

Ray et al

Simonovich et al

121

$10 \quad 40$

$8 \quad 28 \quad 3.7 \%$

$14 \quad 40 \quad 18.5 \%$

$1.08[0.64,1.83]$

$0.11[0.01,2.19]$

$3.82[0.35,41.96]$

$0.47[0.16,1.42]$

$0.49[0.09,2.74]$

$0.59[0.22,1.59]$

$0.47[0.04,5.69]$

$0.13[0.01,1.09]$

$0.62[0.24,1.63]$

$0.95[0.46,1.98]$

$0.64[0.42,0.97]$

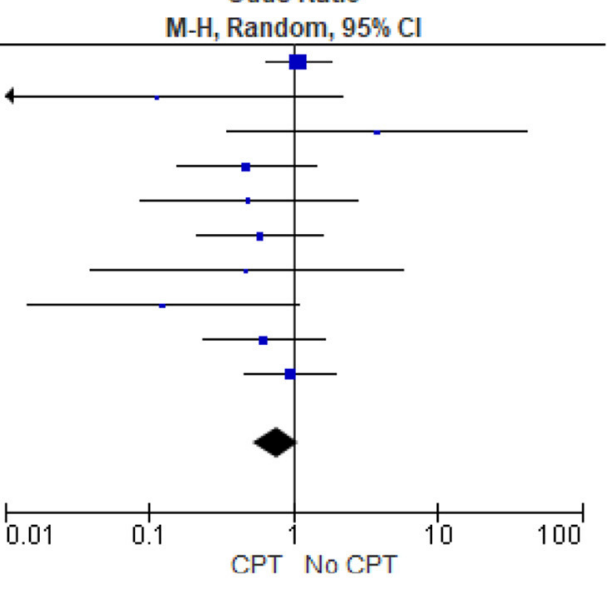

Total $(95 \% \mathrm{Cl})$

535

Total events

56

68

$424 \quad 100.0 \%$

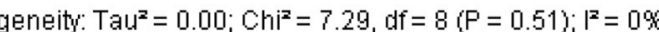

Test for overall effect: $Z=2.10(\mathrm{P}=0.04)$
Odds Ratio

M-H, Random, $95 \% \mathrm{Cl}$

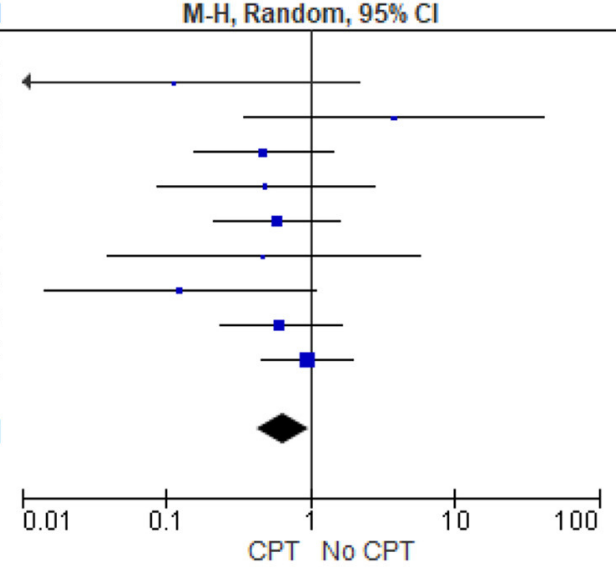

FIGURE 2 | (A) Overall comparison of mortality rate in patients on CPT vs. non-CPT treatment. (B) Subgroup analysis of mortality rate in patients on CPT vs. non-CPT treatment (trial/prospective studies). (C) Subgroup analysis of mortality rate in patients on CPT vs. non-CPT treatment (observational). (D) Sensitivity analysis for mortality rate in patients on CPT vs. non-CPT treatment (trial/prospective studies) true randomized controlled trial (removed pseudorandomized or trial with matched cohort). (E) Sensitivity analysis for mortality rate in patients on CPT vs. non-CPT treatment (trial/prospective studies) true randomized controlled trial [removed Agarwal et al. (28)]. 
TABLE 1B | Study characteristic outcomes.

\begin{tabular}{|c|c|c|c|c|c|c|c|c|c|}
\hline References & $\begin{array}{l}\text { Mortality } \\
\text { CPT arm }\end{array}$ & $\begin{array}{l}\text { Total CPT } \\
\text { patient }\end{array}$ & $\begin{array}{l}\text { Mortality } \\
\text { Non-CPT arm }\end{array}$ & $\begin{array}{l}\text { Total Non-CPT } \\
\text { patient }\end{array}$ & $\begin{array}{l}\text { Adverse event } \\
\text { CPT }\end{array}$ & $\begin{array}{l}\text { ICU } \\
\text { admission }\end{array}$ & ARDS & $\begin{array}{l}\text { Mechanical } \\
\text { ventilation }\end{array}$ & ECMO \\
\hline Abolgashemi et al. (27) & 17 & 115 & 18 & 74 & 1 & N/A & $\mathrm{N} / \mathrm{A}$ & 8 & 0 \\
\hline Ahn et al. (29) & 0 & 2 & & & 0 & 2 & 2 & 2 & 0 \\
\hline Altuntas et al. (54) & 219 & 888 & 245 & 888 & 0 & 21 & $\mathrm{~N} / \mathrm{A}$ & 926 & $\mathrm{~N} / \mathrm{A}$ \\
\hline Avenado-Sola et al. (62) & 0 & 38 & 4 & 43 & 6 & N/A & $\mathrm{N} / \mathrm{A}$ & $\mathrm{N} / \mathrm{A}$ & $\mathrm{N} / \mathrm{A}$ \\
\hline Donato et al. (30) & 11 & 47 & 565 & 1,340 & 1 & $\mathrm{~N} / \mathrm{A}$ & $\mathrm{N} / \mathrm{A}$ & 15 & 0 \\
\hline Duan et al. (11) & 0 & 10 & 3 & 10 & 1 & 2 & 2 & 3 & 0 \\
\hline Gharbaran et al. (31) & 6 & 43 & 11 & 43 & 0 & 31 & 31 & 31 & 5 \\
\hline Liu et al. (35) & 5 & 39 & 38 & 156 & 0 & 4 & 4 & 4 & 0 \\
\hline Hartman et al. (32) & 4 & 31 & & & 0 & 6 & 15 & 10 & 0 \\
\hline Libster et al. (58) & 2 & 80 & 4 & 80 & 0 & 8 & 7 & 6 & 0 \\
\hline Maor et al. (36) & 9 & 49 & & & 1 & & 28 & 28 & 0 \\
\hline Martinez-Resendez et al. (37) & 0 & 8 & & & 0 & 8 & 8 & 5 & 0 \\
\hline Erkurt et al. (52) & 6 & 26 & 0 & 0 & 0 & 0 & 0 & 6 & 0 \\
\hline Olivares-Gazca et al. (38) & 2 & 10 & & & 0 & 10 & 5 & 5 & 0 \\
\hline Omrani et al. (53) & 1 & 40 & 5 & 40 & $\mathrm{~N} / \mathrm{A}$ & 80 & $\mathrm{~N} / \mathrm{A}$ & 69 & 0 \\
\hline Pappa et al. (39) & 0 & 9 & & & 1 & 9 & 9 & 2 & 0 \\
\hline Pei et al. (40) & 0 & 3 & & & 1 & 0 & 0 & 0 & 0 \\
\hline Perotti et al. (41) & 3 & 46 & 7 & 23 & 4 & 16 & 43 & 7 & 2 \\
\hline AlQahtani et al. (60) & 1 & 20 & 2 & 20 & 3 & N/A & $\mathrm{N} / \mathrm{A}$ & 10 & 0 \\
\hline Wang et al. (46) & 3 & 5 & & & 0 & 5 & 5 & 5 & 0 \\
\hline Xia et al. (47) & 3 & 138 & 59 & 1,430 & 0 & 3 & 22 & 28 & 2 \\
\hline Ye et al. (48) & 0 & 6 & & & 0 & 1 & 0 & 4 & 0 \\
\hline Yoon et al. (57) & 23 & 73 & 28 & 73 & 0 & N/A & N/A & 18 & 0 \\
\hline Zeng et al. (49) & 5 & 6 & 14 & 15 & 0 & 6 & 6 & 5 & 1 \\
\hline Zhang et al. (50) & 0 & 4 & & & 0 & 4 & 4 & 2 & 2 \\
\hline
\end{tabular}

N/A, not available/not mentioned.

all the authors, and decisions were made via a consensus. The Cochrane tool for risk of bias (19) was used for RCTs (Table 2A), and the correlation of quality measures with estimates of treatment effects in meta-analyses of RCTs (20) was used for quality assessment of the same (Table 2B). Non-randomized studies were evaluated using the NOS for the case-control/cohort (22) (Tables 2C,D) and the NIH Quality Assessment Tool for Case Series Studies (21) (Table 2E). Quality assessments were conducted independently, and discrepancies were resolved by consensus. Overall, risk-of-bias assessment showed that the included studies had low to medium risk of bias.

\section{DISCUSSION}

In this systematic review and meta-analysis of CPT in COVID19 patients, 38 studies (11, 12, 27-62) were included and critically evaluated. All included studies reported excellent outcomes for CPT in COVID-19. Our systemic review and meta-analysis is one of the first ones to summarize all such existing evidence on the efficacy and safety of CPT in humans with COVID-19. According to the results of our systematic review and meta-analysis, $\mathrm{CPT}$ is effective in reducing the mortality rate and has low incidence of serious adverse events 


\section{Pooled mortality rate for patients with CPT in COVID-19}

\begin{tabular}{|c|c|c|c|}
\hline \multirow[t]{2}{*}{ Study name } & \multicolumn{3}{|c|}{ Statistio for each s tudy } \\
\hline & $\begin{array}{l}\text { Event } \\
\text { rate }\end{array}$ & $\begin{array}{l}\text { Lower } \\
\text { limit }\end{array}$ & $\begin{array}{l}\text { Upper } \\
\text { limit }\end{array}$ \\
\hline Abalgashemiet al & 0.148 & 0.094 & 0.225 \\
\hline Agawalet al & 0.145 & 0.105 & 0.190 \\
\hline Ahnet a & 0.167 & 0.010 & $0.80 \mathrm{C}$ \\
\hline Atuntas etal & 0.247 & 0.219 & 0.270 \\
\hline Avendano-Sob et al & 0.013 & 0.001 & 0.175 \\
\hline Bajpsiet al & 0.214 & 0.071 & 0.494 \\
\hline Donata et al & 0.234 & 0.135 & 0.375 \\
\hline Dusnet al & 0.045 & 0.003 & 0.443 \\
\hline Ghatbaenet al & 0.140 & 0.064 & 0.278 \\
\hline Hifuet af & 0.128 & 0.054 & 0.273 \\
\hline Hartmanet a & 0.129 & 0.049 & 0.297 \\
\hline Hegerones al a & 0.100 & 0.025 & 0.324 \\
\hline Jopneret al & 0.085 & 0.082 & 0.090 \\
\hline Karekadaroth et al & 0.100 & 0.006 & 0.674 \\
\hline Lasteret al & 0.025 & 0.005 & 0.094 \\
\hline Ling Liet al & 0.157 & 0.080 & 0.284 \\
\hline Msor et al & 0.184 & 0.098 & 0.317 \\
\hline Martinetresender a al & 0.056 & 0.003 & 0.505 \\
\hline Mehmetet al & 0.231 & 0.108 & 0.428 \\
\hline Olivares-ganas et a & 0.200 & 0.050 & 0.541 \\
\hline Omraniet al & 0.025 & 0.004 & 0.157 \\
\hline Pappoet al & 0.050 & 0,003 & 0.475 \\
\hline Peietal & 0.125 & 0.007 & 0.734 \\
\hline Perctti a al & 0.065 & 0.021 & 0.184 \\
\hline Quhbaniet al & 0.050 & 0.007 & 0.282 \\
\hline Rashoed et al & 0.048 & 0.007 & 0.271 \\
\hline Rayetal & 0.250 & 0.140 & 0.405 \\
\hline Rogess et al & 0.125 & 0.064 & 0.231 \\
\hline Salazaret al & 0.037 & 0.015 & 0.085 \\
\hline Shen et al & 0.083 & 0.005 & 0.622 \\
\hline Simonorich et al & 0.110 & 0.075 & 0.157 \\
\hline Tanetal & 0.167 & 0.010 & 0.500 \\
\hline Warg et al & 0.600 & 0.200 & 0.900 \\
\hline Ximpl xiset al & 0.022 & 0.007 & 0.055 \\
\hline Yeetal & 0.071 & 0.004 & 0.577 \\
\hline Yoonet al & 0.315 & 0.219 & 0.430 \\
\hline Zeng etal & 0.833 & 0.369 & 0.977 \\
\hline \multirow[t]{2}{*}{ Zharg thet al } & 0.100 & 0,005 & 0.674 \\
\hline & 0.129 & 0.097 & 0.109 \\
\hline
\end{tabular}

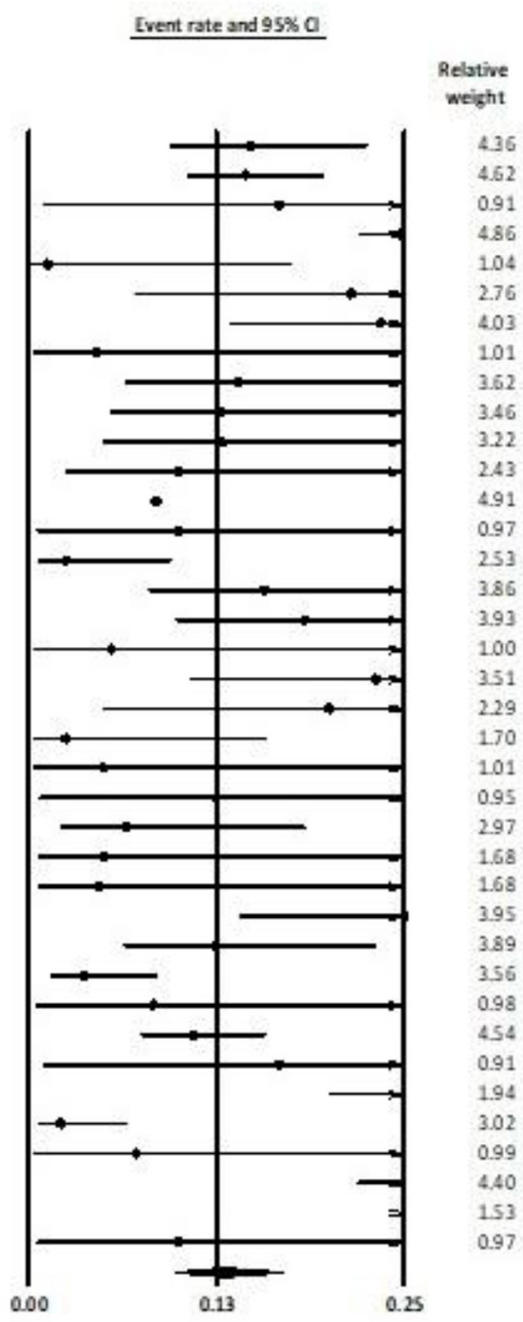

FIGURE 3 | Pooled mortality rate with use of CPT in COVID-19.

during and after convalescent plasma infusion, which are mostly controllable.

CPT confers immediate immunity via interruption of the viral entry into the cells. Additionally, in the context of COVID-19, neutralizing antibodies are anticipated to be the primary active agent in convalescent plasma and the marker of plasma potency (9). In the past, CPT has been shown to provide benefits in severe acute respiratory syndromes (6). Prior studies have also reported promising outcomes in Spanish influenza A (H1N1) infection (63), avian influenza A (H5N1) (64), viral hemorrhagic fevers such as Ebola (65), influenza A (H1N1) infections in 2009/2010 (66), and SARS-CoV infections in 2003 (67). A systematic review and meta-analysis revealed a consistent reduction in mortality with the use of plasma therapy (6). The results are similar to our findings. One of the possible hypotheses for the observed decreased mortality could be due to antibodies that can hamper virus reproduction in the active phase of infection and help clear the virus, which is advantageous to the rapid recovery of the disease (67). Mechanistic and clinical data also support the observed mortality reduction benefit associated with convalescent plasma administration $(68,69)$.

There was no significant reduction in mortality rate between patients with CPT and controls based on data from RCTs. However, sensitivity analysis [excluding the study by Agarwal et al. (28)] revealed that patients transfused with CPT had a lower mortality rate. The Agarwal et al. (28) trial comprised 


\section{Pooled adverse event rate for CPT in COVID-19}

\begin{tabular}{|c|c|c|c|}
\hline \multirow[t]{3}{*}{ Studyname } & \multicolumn{3}{|c|}{ Statistic for each s tudy } \\
\hline & Event: & Lower & Upper \\
\hline & rate & limit & limit \\
\hline Abolgashemi et al & 0.009 & 0.001 & 0.059 \\
\hline Agarmal et al & 0.038 & 0.020 & 0.072 \\
\hline Ahn et al & 0.167 & 0.010 & 0.805 \\
\hline Avendano-Sols et af & 0.158 & 0.073 & 0.310 \\
\hline Bajpsi et al & 0.071 & 0.010 & 0.370 \\
\hline Donsto et al & 0.021 & 0.003 & 0.136 \\
\hline Duanet al & 0.100 & 0.014 & 0.457 \\
\hline Gharbaran et al & 0.011 & 0.001 & 0.157 \\
\hline H.jud etal & 0.013 & 0.001 & 0.171 \\
\hline Hortmon etal & 0.016 & 0.001 & 0.206 \\
\hline Hegerora et al & 0.200 & 0.077 & 0.428 \\
\hline Jopneret al & 0.054 & 0.051 & 0.068 \\
\hline Karekadave thet al & 0.100 & 0.005 & 0.574 \\
\hline Libsteretal & 0,005 & 0.000 & 0.091 \\
\hline Ling bietal & 0.039 & 0.010 & 0.144 \\
\hline Maoret al & 0.020 & 0.003 & 0.131 \\
\hline Martiner-resendet & 0.056 & 0.003 & 0.505 \\
\hline Mehmetet al & 0.019 & 0.001 & 0.236 \\
\hline Olivares ganas et al & 0.045 & 0.003 & 0.448 \\
\hline Omrani et al & 0.775 & 0.521 & 0.879 \\
\hline Pappa et al & 0.111 & 0.015 & 0.500 \\
\hline Peiet al & 0.333 & 0.043 & 0.845 \\
\hline Perottietal & 0.087 & 0.033 & 0.210 \\
\hline Qathani et al & 0.150 & 0.049 & 0.376 \\
\hline Rastreed et al & 0.095 & 0.024 & 0.311 \\
\hline Raret al & 0.012 & 0.001 & 0.167 \\
\hline Rogersetal & 0.047 & 0.015 & 0.135 \\
\hline Salazar etal & 0.004 & 0.000 & 0.056 \\
\hline Shenetal & 0.083 & 0.005 & 0.622 \\
\hline Simonorich et al & 0.671 & 0.607 & 0.729 \\
\hline Tan etal & 0.167 & 0.010 & 0.805 \\
\hline Wanget al & 0.083 & 0.005 & 0.622 \\
\hline Xinylxis et al & 0.004 & 0.000 & 0.055 \\
\hline Yeet al & 0.071 & 0.004 & 0.577 \\
\hline Yoon et al & 0.007 & 0.000 & 0.099 \\
\hline Zenget al & 0.071 & 0.004 & 0.577 \\
\hline \multirow[t]{2}{*}{ Zhargh betal } & 0.100 & 0.005 & 0.674 \\
\hline & 0.051 & 0.032 & 0.116 \\
\hline
\end{tabular}

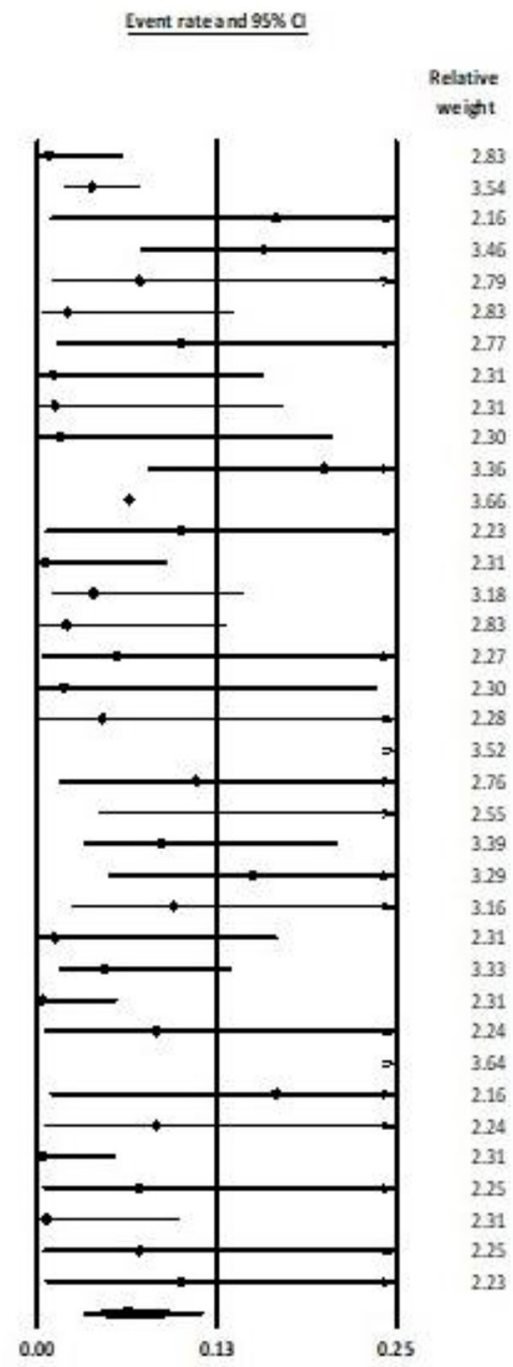

FIGURE 4 | Pooled adverse event rate with use of CPT in COVID-19.

$\sim 70 \%$ of the patients in the CPT cohort who received plasma with low levels of SARS-CoV-2 antibodies. Additionally, the remaining $30 \%$ of the patients received plasma with no detectable antibodies. Thus, there were strong methodical and clinical rationales to exclude this study from statistical models during sensitivity analysis. Nevertheless, Agarwal et al. (28) did observe a positive effect of CPT on clinical symptoms and viral clearance.

It is worth noting that the doses of CPT vary between the included studies. However, the Chinese study (11) described the use of a single dose of $200 \mathrm{ml}$ of convalescent plasma, whereas Bin Zhang et al. (50) reported a maximum of 2,400 $\mathrm{ml}$ of convalescent plasma. The optimal dose of CPT for COVID-19, therefore, could not be estimated.
It is also important to note that the included patients were critically ill and received ICU admission $(n=12,095)$ or underwent mechanical ventilation $(n=8,200)$ and that all COVID-19 patients described in our meta-analysis received concomitant antiviral drugs and steroids including CPT; also, many patients received antibacterial/antifungal drugs for coinfection. All included studies described little mortality with the use of CPT, and the pooled analysis suggests a mortality rate of $12.9 \%$ (95\% CI 9.7-16.9). However, the individual impact of CPT could not be determined as patients also received multiple other agents (including antiviral medications). Therefore, further studies evaluating the use of CPT alone are warranted.

The safety profile of CPT in COVID-19 has not been described in detail. The observed pooled adverse event rate was $6.1 \%$ 
TABLE 2A | Assessment of the trials included in the study.

\begin{tabular}{|c|c|c|c|c|c|c|c|c|c|c|}
\hline References & $\begin{array}{l}\text { Abolgashemi } \\
\text { et al. (27) }\end{array}$ & $\begin{array}{l}\text { Agarwal } \\
\text { et al. } \\
\text { (28) }\end{array}$ & $\begin{array}{l}\text { AlQahtani } \\
\text { et al. }(60)\end{array}$ & $\begin{array}{l}\text { Avenado-Sola } \\
\text { et al. (62) }\end{array}$ & $\begin{array}{l}\text { Bajpai } \\
\text { et al. } \\
\text { (59) }\end{array}$ & $\begin{array}{l}\text { Donato } \\
\text { et al. }(30)\end{array}$ & $\begin{array}{l}\text { Erkurt } \\
\text { et al. } \\
\text { (52) }\end{array}$ & $\begin{array}{l}\text { Hartman } \\
\text { et al. (32) }\end{array}$ & $\begin{array}{l}\text { Gharbaran } \\
\text { et al. (31) }\end{array}$ & $\begin{array}{l}\text { Joyner } \\
\text { et al. (12) }\end{array}$ \\
\hline $\begin{array}{l}\text { Study question well-defined in } \\
\text { introduction/methods }\end{array}$ & Yes & Yes & Yes & Yes & No & Yes & Yes & Yes & Yes & Yes \\
\hline $\begin{array}{l}\text { Study question well-defined } \\
\text { anywhere in the article }\end{array}$ & Yes & Yes & Yes & Yes & No & Yes & Yes & Yes & Yes & Yes \\
\hline Placebo control & No & No & No & No & No & No & No & No & No & No \\
\hline Appropriate outcome studied & Yes & Yes & Yes & Yes & Yes & Yes & Yes & Yes & Yes & Yes \\
\hline Multicenter study & Yes & Yes & Yes & Yes & No & No & No & No & Yes & Yes \\
\hline Study country & Iran & India & Bahrain & Spain & India & USA & Turkey & USA & Netherlands & USA \\
\hline Adequate selection criteria & Yes & Yes & Yes & Yes & Yes & Yes & No & Yes & Yes & Yes \\
\hline Randomization methods described & N/A & Yes & Yes & Yes & Yes & $\mathrm{N} / \mathrm{A}$ & N/A & N/A & Yes & N/A \\
\hline Central randomization site & N/A & Yes & $\mathrm{N} / \mathrm{D}$ & $N / D$ & N/A & N/A & N/A & N/A & $N / D$ & $\mathrm{~N} / \mathrm{A}$ \\
\hline Allocation concealment & $N / D$ & Yes & No & Yes & Yes & $N / D$ & N/A & $N / D$ & No & $N / D$ \\
\hline Patients blinded & N/D & No & No & No & No & $N / D$ & N/A & N/D & No & $N / D$ \\
\hline Caregivers blinded & N/D & No & No & No & No & $N / D$ & N/A & N/D & No & $N / D$ \\
\hline Outcome assessors blinded & N/D & No & $\mathrm{N} / \mathrm{D}$ & N/D & N/D & $N / D$ & $\mathrm{~N} / \mathrm{A}$ & N/D & N/D & $\mathrm{N} / \mathrm{D}$ \\
\hline Data analysts blinded & $N / D$ & $N / D$ & $N / D$ & N/D & $N / D$ & $N / D$ & N/A & N/D & $N / D$ & $N / D$ \\
\hline Double blinded & N/D & No & No & No & No & $N / D$ & $\mathrm{~N} / \mathrm{A}$ & N/D & $\mathrm{N} / \mathrm{D}$ & $\mathrm{N} / \mathrm{D}$ \\
\hline Vital statistical measures & Yes & Yes & Yes & Yes & Yes & Yes & Yes & Yes & Yes & Yes \\
\hline Statistician author or acknowledged & No & Yes & No & Yes & $N / D$ & Yes & No & No & No & Yes \\
\hline Intention-to-treat analysis & No & Yes & No & No & No & No & No & No & No & No \\
\hline Power calculation reported & No & Yes & Yes & Yes & $N / D$ & Yes & No & No & Yes & No \\
\hline Stopping rules described & No & No & No & Yes & $N / D$ & No & No & No & Yes & No \\
\hline Baseline characteristics reported & Yes & Yes & Yes & Yes & Yes & Yes & Yes & Yes & Yes & Yes \\
\hline Groups similar at baseline & No & Yes & Yes & $N / D$ & Yes & N/A & N/A & No & No & N/A \\
\hline Confounders accounted for & Yes & Yes & Yes & No & Yes & Yes & No & Yes & Yes & No \\
\hline Percentage dropouts & N/A & Yes & $\mathrm{N} / \mathrm{A}$ & Yes & Yes & N/A & N/A & N/A & N/A & $\mathrm{N} / \mathrm{A}$ \\
\hline Reasons for dropout given & N/A & Yes & N/A & Yes & Yes & N/A & $\mathrm{N} / \mathrm{A}$ & N/A & $\mathrm{N} / \mathrm{A}$ & N/A \\
\hline Findings support conclusion & Yes & Yes & Yes & Yes & Yes & Yes & Yes & Yes & Yes & Yes \\
\hline
\end{tabular}


TABLE 2A | Continued

\begin{tabular}{|c|c|c|c|c|c|c|c|c|c|}
\hline References & Li et al. (34) & $\begin{array}{l}\text { Libster } \\
\text { et al. (58) }\end{array}$ & $\begin{array}{l}\text { Olivares-Gazca } \\
\text { et al. (38) }\end{array}$ & $\begin{array}{l}\text { Pappa } \\
\text { et al. (39) }\end{array}$ & $\begin{array}{l}\text { Perotti } \\
\text { et al. (41) }\end{array}$ & $\begin{array}{l}\text { Rasheed } \\
\text { et al. (42) }\end{array}$ & $\begin{array}{l}\text { Ray et al. } \\
\text { (61) }\end{array}$ & $\begin{array}{l}\text { Salazar } \\
\text { et al. (43) }\end{array}$ & $\begin{array}{l}\text { Simonovich } \\
\text { et al. (56) }\end{array}$ \\
\hline $\begin{array}{l}\text { Study question well-defined in } \\
\text { introduction/methods }\end{array}$ & Yes & Yes & Yes & Yes & Yes & Yes & Yes & Yes & Yes \\
\hline $\begin{array}{l}\text { Study question well-defined } \\
\text { anywhere in the article }\end{array}$ & Yes & Yes & Yes & Yes & Yes & Yes & Yes & Yes & Yes \\
\hline Placebo control & No & Yes & No & No & No & No & No & No & Yes \\
\hline Appropriate outcome studied & Yes & Yes & Yes & Yes & Yes & Yes & Yes & Yes & Yes \\
\hline Multicenter study & Yes & Yes & No & Yes & Yes & Yes & No & Yes & Yes \\
\hline Study country & China & Argentina & Mexico & Greece & Italy & Iraq & India & USA & Argentina \\
\hline Adequate selection criteria & Yes & Yes & Yes & Yes & Yes & Yes & Yes & Yes & Yes \\
\hline Randomization methods described & Yes & Yes & N/A & N/A & No & Yes & No & N/A & Yes \\
\hline Central randomization site & Yes & Yes & $\mathrm{N} / \mathrm{A}$ & N/A & No & No & $N / D$ & N/A & $N / D$ \\
\hline Allocation concealment & N/D & Yes & $N / D$ & $N / D$ & $N / D$ & $N / D$ & No & $N / D$ & Yes \\
\hline Patients blinded & N/D & Yes & $N / D$ & $N / D$ & $N / D$ & $N / D$ & No & $N / D$ & Yes \\
\hline Caregivers blinded & N/D & Yes & $N / D$ & $N / D$ & $N / D$ & $N / D$ & No & $N / D$ & Yes \\
\hline Outcome assessors blinded & N/D & $N / D$ & $\mathrm{~N} / \mathrm{D}$ & $N / D$ & $N / D$ & $N / D$ & $N / D$ & N/D & Yes \\
\hline Data analysts blinded & $N / D$ & $N / D$ & $N / D$ & $N / D$ & $N / D$ & $N / D$ & $N / D$ & $N / D$ & No \\
\hline Double blinded & N/D & Yes & $N / D$ & $N / D$ & $N / D$ & N/D & No & $N / D$ & Yes \\
\hline Vital statistical measures & No & Yes & No & Yes & No & No & Yes & Yes & Yes \\
\hline Statistician author or acknowledged & Yes & $N / D$ & No & No & Yes & No & No & No & Yes \\
\hline Intention-to-treat analysis & Yes & Yes & No & No & Yes & No & No & No & Yes \\
\hline Power calculation reported & No & Yes & No & No & No & No & No & No & Yes \\
\hline Stopping rules described & No & Yes & No & No & No & No & No & No & No \\
\hline Baseline characteristics reported & Yes & Yes & Yes & Yes & Yes & Yes & Yes & Yes & Yes \\
\hline Groups similar at baseline & Yes & No & N/A & N/A & Yes & Yes & $N / D$ & Yes & No \\
\hline Confounders accounted for & Yes & Yes & Yes & Yes & Yes & No & No & Yes & Yes \\
\hline Percentage dropouts & N/A & Yes & N/A & N/A & N/A & N/A & $N / A$ & $\mathrm{~N} / \mathrm{A}$ & $N / A$ \\
\hline Reasons for dropout given & N/A & Yes & N/A & N/A & N/A & N/A & $N / A$ & N/A & $N / A$ \\
\hline Findings support conclusion & Yes & Yes & Yes & Yes & Yes & Yes & Yes & Yes & Yes \\
\hline
\end{tabular}

N/A, not available/not applicable; N/D, not defined. 
TABLE 2B | Risk-of-bias assessment of the trials included in the study.

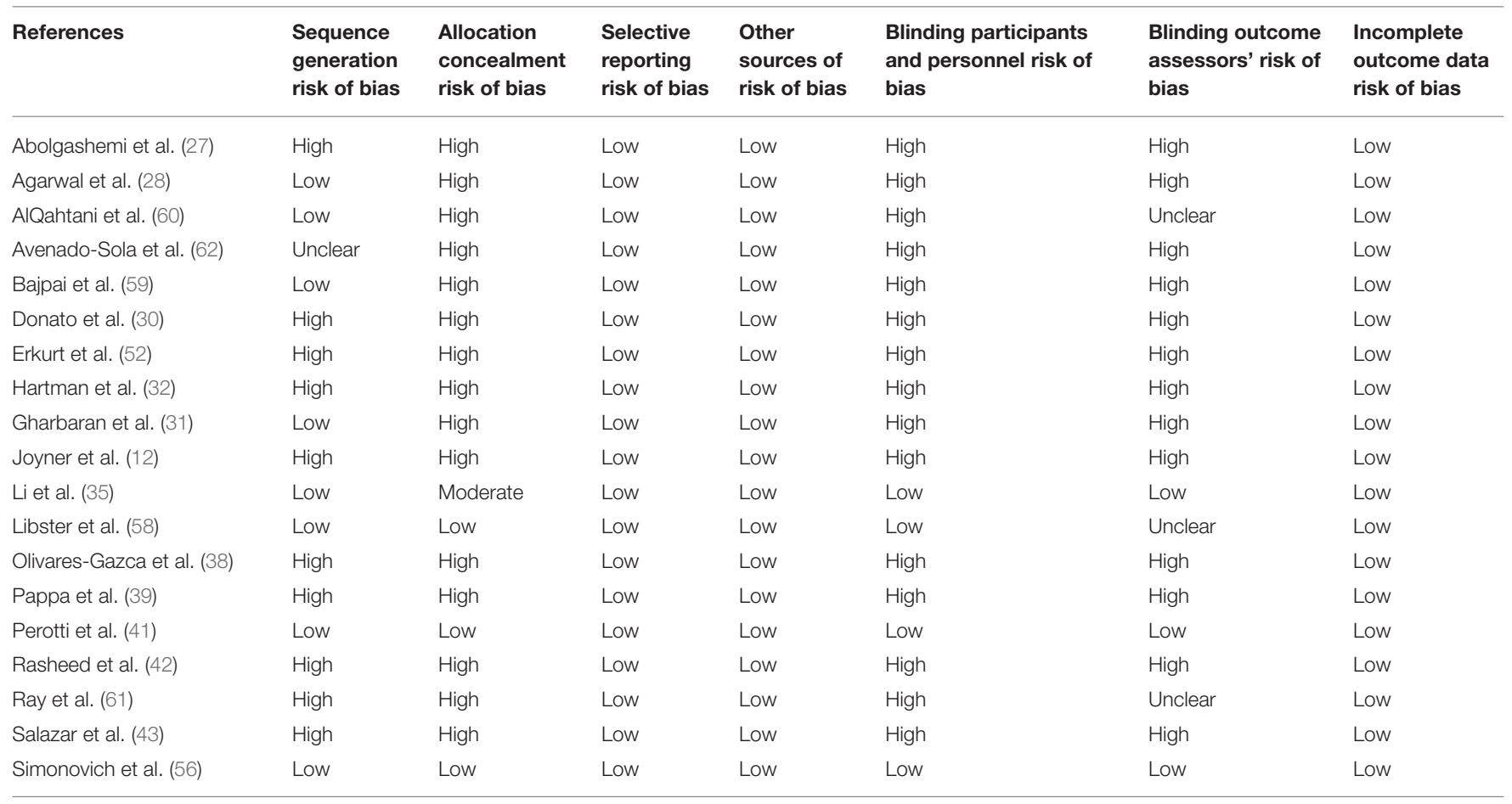

TABLE 2C | Quality assessment for case-control studies included in the study.

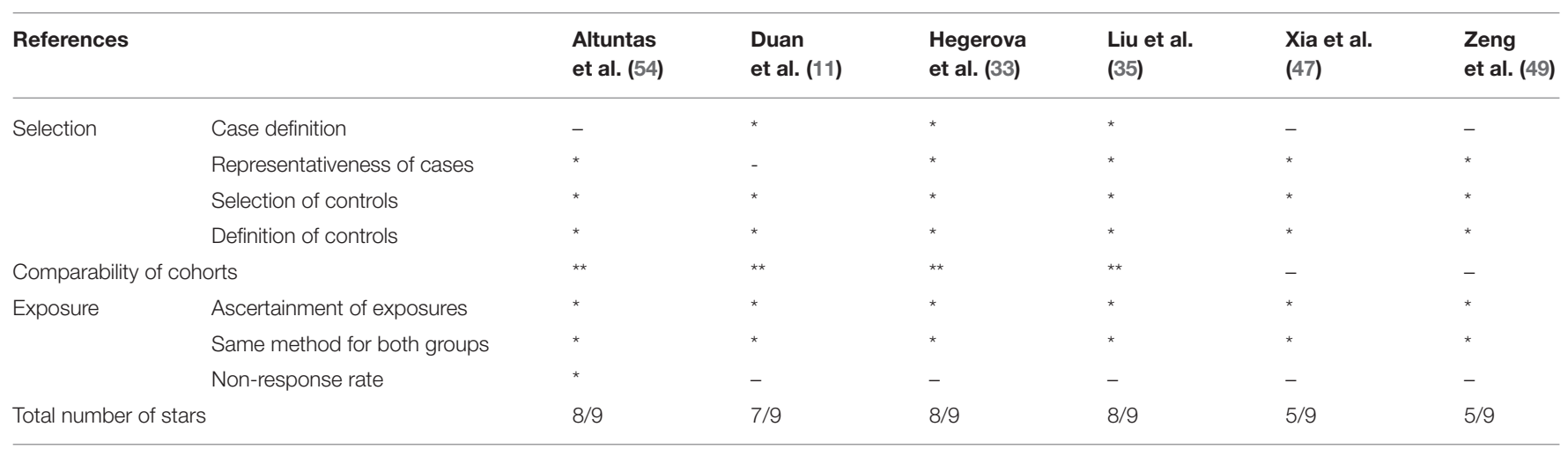

Note: A study can be awarded a maximum of one star for each numbered item within the Selection and Exposure categories. A maximum of two stars can be given for Comparability.

TABLE 2D | Quality assessment for cohort studies included in the study.

\begin{tabular}{|c|c|c|c|c|c|}
\hline References & & Maor et al. (36) & Omrani et al. (53) & Rogers et al. (55) & Yoon et al. (57) \\
\hline \multirow[t]{3}{*}{ Selection } & Representativeness of cohort & * & * & * & * \\
\hline & Selection of non-exposed cohort & - & * & * & * \\
\hline & Outcome not present at the beginning & - & * & * & * \\
\hline Comparability of cohorts & & - & ** & ** & ** \\
\hline & Adequacy of follow-up & * & * & * & * \\
\hline Total number of stars & & $4 / 9$ & 9/9 & $8 / 9$ & $9 / 9$ \\
\hline
\end{tabular}

Note: A study can be awarded a maximum of one star for each numbered item within the Selection and Exposure categories. A maximum of two stars can be given for Comparability. 
TABLE 2E | Quality assessment of the case studies included in the study.

\begin{tabular}{|c|c|c|c|c|c|c|c|c|c|}
\hline References & $\begin{array}{l}\text { Ahn et al. } \\
\text { (29) }\end{array}$ & $\begin{array}{l}\text { Karekadavath } \\
\text { et al. (51) }\end{array}$ & $\begin{array}{l}\text { Martinez-Resendez } \\
\text { et al. (37) }\end{array}$ & $\begin{array}{l}\text { Pei et al. } \\
(40)\end{array}$ & $\begin{array}{l}\text { Shen } \\
\text { et al. (44) }\end{array}$ & $\begin{array}{l}\text { Tan } \\
\text { et al. } \\
(45)\end{array}$ & $\begin{array}{l}\text { Wang } \\
\text { et al. (46) }\end{array}$ & $\begin{array}{l}\text { Ye et al. } \\
(48)\end{array}$ & $\begin{array}{l}\text { Zhang } \\
\text { et al. (50) }\end{array}$ \\
\hline $\begin{array}{l}\text { 1. Was the study question or objective } \\
\text { clearly stated? }\end{array}$ & Yes & Yes & Yes & Yes & Yes & Yes & Yes & Yes & Yes \\
\hline $\begin{array}{l}\text { 2. Was the study population clearly and } \\
\text { fully described, including a case definition? }\end{array}$ & Yes & No & Yes & Yes & Yes & Yes & No & Yes & Yes \\
\hline 3. Were the cases consecutive? & No & Yes & $N / D$ & N/D & No & $N / D$ & N/D & No & No \\
\hline 4. Were the subjects comparable? & Yes & Yes & Yes & Yes & Yes & No & Yes & No & Yes \\
\hline 5. Was the intervention clearly described? & Yes & Yes & Yes & No & Yes & No & Yes & Yes & Yes \\
\hline $\begin{array}{l}\text { 6. Were the outcome measures clearly } \\
\text { defined, valid, reliable, and implemented } \\
\text { consistently across all study participants? }\end{array}$ & Yes & Yes & Yes & No & Yes & No & Yes & Yes & Yes \\
\hline 7. Was the length of follow-up adequate? & Yes & Yes & No & Yes & Yes & No & No & No & Yes \\
\hline $\begin{array}{l}\text { 8. Were the statistical methods } \\
\text { well-described? }\end{array}$ & $\mathrm{N} / \mathrm{A}$ & N/A & Yes & N/A & $\mathrm{N} / \mathrm{A}$ & $\mathrm{N} / \mathrm{A}$ & Yes & $\mathrm{N} / \mathrm{A}$ & $\mathrm{N} / \mathrm{A}$ \\
\hline 9. Were the results well-described? & Yes & Yes & Yes & Yes & Yes & Yes & Yes & Yes & Yes \\
\hline Quality rating & Good & Good & Good & Fair & Good & Poor & Fair & Fair & Good \\
\hline
\end{tabular}

N/A, not applicable; N/D, not defined.

(95\% CI 3.2-11.6). This suggests that CPT was well-tolerated by the participants in the included studies. It is important to note that no fatality was reported as adverse event with the use of CPT. Human plasma transfusion is routinely performed in hospitals. Human anti-SARS-CoV-2 plasma differs from standard plasma as it contains antibodies against SARS-CoV-2. The risks to transfusion recipients are similar to those of standard plasma. The risk of transfusion-transmissible infection is low in developed countries. The incidence rates of infections such as HIV, hepatitis B, and hepatitis $\mathrm{C}$ are less than one infection per 2 million donations (70). Other adverse events with plasma therapy include allergic transfusion reactions, transfusion-associated circulatory overload (TACO), and transfusion-related acute lung injury (TRALI) (71). Even though TRALI occurs in $<1$ for every 5,000 transfused units, it is concerning in COVID-19 patients. Donor screening including HLA antibody screening decreases the risk of TRALI (72).

A risk benefit analysis based on age, symptoms, comorbidities, and COVID-19 transmission parameters was published in a recent review by Bloch et al. (73). Five hundred simulations were carried out, assuming varying degrees of effectiveness of convalescent plasma treatment. The model revealed that convalescent plasma was beneficial in COVID-19 infection even at the lowest estimates of $25 \%$ effectiveness. In other words, the model suggests that the potential benefit of plasma therapy outweighs the risk of transfusion in COVID-19 infection (73).

The important strengths of our study are a comprehensive search of the already published clinical studies and the large number of patients included in the analysis. This is one of the first meta-analyses on CPT use in COVID-19 patients showing an overwhelming positive result. The review of sample of articles by two co-authors is again a testimony of the quality check of data collection in this review. The generalizability of these results is also a strength of this article.
Despite the numerous strengths of the meta-analysis, there are certain limitations. One of the limitations of the meta-analysis is integral to the methodology. The summarization of varying pieces of information may ignore important differences between studies. Nonetheless, this is a controversial aspect of the metaanalysis (74). Additionally, a meta-analysis generalizes results despite differences in primary research and does not simply report a summary effect. The heterogeneity is high in our studies, especially regarding the pooled adverse event rate and pooled mortality rate. Further studies may be needed to confirm the findings and explain the mechanisms. A lack of high-quality RCT studies and relevant literature paucity limited our analyses. All the reported studies were predominately case reports or series, had no proper control groups, and had a moderate to high risk of bias. Most studies in our meta-analysis were observational studies with a high risk of bias, which are subject to inherent limitations of the study design with unmeasured differences in the study population and residual confounders despite all adjustments. The currently available evidence on the safety and effectiveness of convalescent plasma for treatment of people with COVID-19 is of very low strength. Our study predominantly describes the clinical data and incidence rates in hospitalized patients. Also, we could not register the review. We tried to prospectively register our systematic review but decided to go against it as it was taking an unreasonably longer time than expected due to the increased pool of COVID-19-related articles. Another limitation of our study is the inclusion of 12 studies $(30,31,37,39,40,42,45,57-59,61,62)$ from the preprint databases which have not been peer reviewed and are necessary for a thorough evaluation of the currently available data on CPT in our meta-analysis. Preprint articles possibly indicate the undetermined quality of available literature and biased articles on CPT; however, we will update the status of these abovementioned studies in the risk-of-bias table. Lastly, many studies were determined to have a significant risk of bias. This was 
related to a combination of factors such as non-randomized design, confounding, poor methodological conduct, and limited information on dose and duration of the CPT. Importantly, many of the patients enrolled in the studies included in the present analysis received convalescent plasma transfusions later in their disease course. As a result, our analysis may underestimate the mortality reduction achievable through early administration of high-titer convalescent plasma for COVID-19. Based on low-quality evidence, there is no suggestion that convalescent plasma would cause any serious adverse events in patients with COVID-19 and lower the mortality in COVID-19 patients. Thus, any conclusions that are drawn based on these data are of limited value, and these conclusions are subject to change as more reliable results become available.

\section{CONCLUSION}

Based on the consolidated clinical data derived from the systemic review and meta-analysis, it is suggested that, in addition to antiviral/antimicrobial drugs and steroids, CPT could be an effective concomitant therapeutic option as the use of CPT decreased mortality with a safe clinical profile and promising evidence on the safety and reduced mortality. We recognize that a definitive conclusion cannot be drawn

\section{REFERENCES}

1. Shah A, Kashyap R, Tosh P, Sampathkumar P, O'Horo JC. Guide to understanding the 2019 novel coronavirus. Mayo Clin Proc. (2020) 95:646-52. doi: 10.1016/j.mayocp.2020.02.003

2. Cucinotta D, Vanelli M. WHO declares COVID-19 a pandemic. Acta Biomed. (2020) 91:157-60. doi: 10.23750/abm.v91i1.9397

3. World Health Organization. Report of the WHO-China Joint Mission on Coronavirus Disease 2019 (COVID-19). Geneva (2020). https://www.who.int/ docs/default-source/coronaviruse/who-china-joint-mission-on-covid-19final-report.

4. Dong E, Du H, Gardner L. An interactive web-based dashboard to track COVID-19 in real time. Lancet Infect Dis. (2020) 20:533-4. doi: 10.1016/S1473-3099(20)30120-1

5. BMJ Best Practice US. Coronavirus Disease 2019 (COVID-19) - Complications. (2020). Available online at: https://bestpractice.bmj.com/topics/en-us/ 3000168/complications (accessed January 18, 2021).

6. Mair-Jenkins J, Saavedra-Campos M, Baillie JK, Cleary P, Khaw FM, Lim WS, et al. The effectiveness of convalescent plasma and hyperimmune immunoglobulin for the treatment of severe acute respiratory infections of viral etiology: a systematic review and exploratory meta-analysis. J Infect Dis. (2015) 211:80-90. doi: 10.1093/infdis/jiu396

7. van Griensven J, De Weiggheleire A, Delamou A, Smith PG, Edwards T, Vandekerckhove $\mathrm{P}$, et al. The use of ebola convalescent plasma to treat ebola virus disease in resource-constrained settings: a perspective from the field. Clin Infect Dis. (2016) 62:69-74. doi: 10.1093/cid/civ680

8. Andreano E, Piccini G, Licastro D, Casalino L, Johnson NV, Paciello I, et al. SARS-CoV-2 escape in vitro from a highly neutralizing COVID-19 convalescent plasma. bioRxiv [Preprint]. (2020). doi: $10.1101 / 2020.12 .28 .424451$

9. Rojas M, Rodríguez Y, Monsalve DM, Acosta-Ampudia Y, Camacho B, Gallo JE, et al. Convalescent plasma in Covid-19: possible mechanisms of action. Autoimmun Rev. (2020) 19:102554. doi: 10.1016/j.autrev.2020.102554

10. U. S. Food Drug Administration. Investigational COVID-19 Convalescent Plasma-Emergency INDs. (2020). Available online at: https://www.fda. gov/vaccines-blood-biologics/investigational-new-drug-ind-or-device- regarding optimal doses and treatment time point for the CPT. Future larger observational studies (75) and clinical trials could benefit from more standardized reporting, especially in terms of the timing of intervention and clinically relevant outcomes, like days until discharge from hospital and improvement of clinical symptoms.

\section{DATA AVAILABILITY STATEMENT}

The raw data supporting the conclusions of this article will be made available by the authors, without undue reservation.

\section{AUTHOR CONTRIBUTIONS}

Data review and collection were done by KM, IG, SG, RS, AT, PA, $\mathrm{HK}$, and SD. Statistical analysis was done by VB, AB, and MS. Study design and distribution of articles for critical review were done by IM and RK. VB and KM are the guarantors of the paper, taking responsibility for the integrity of the work as a whole, and from inception to publication of the article. Final approval was given by all authors. All authors contributed to the article and approved the submitted version.

exemption-ide-process-cber/recommendations-investigational-covid-19convalescent-plasma. Published (accessed January 31, 2021).

11. Duan K, Liu B, Li C, Zhang H, Yu T, Qu J, et al. Effectiveness of convalescent plasma therapy in severe COVID-19 patients. Proc Natl Acad Sci USA. (2020) 117:9490-6. doi: 10.1073/pnas.2004168117

12. Joyner MJ, Bruno KA, Klassen SA, Kunze KL, Johnson PW, Lesser ER, et al. Safety update: COVID-19 convalescent plasma in 20,000 hospitalized patients. Mayo Clin Proc. (2020) 95:1888-97. doi: 10.1016/j.mayocp.2020. 09.032

13. Roberts DJ, Miflin G, Estcourt L. Convalescent plasma for COVID-19: back to the future. Transfus Med. (2020) 30:174-6. doi: 10.1111/tme.12700

14. Valk SJ, Piechotta V, Chai KL, Doree C, Monsef I, Wood EM, et al. Convalescent plasma or hyperimmune immunoglobulin for people with COVID-19: a rapid review. Cochrane Datab Syst Rev. (2020) 5:Cd013600. doi: 10.1002/14651858.CD013600

15. Piechotta V, Chai KL, Valk SJ, Doree C, Monsef I, Wood EM, et al. Convalescent plasma or hyperimmune immunoglobulin for people with COVID-19: a living systematic review. Cochrane Datab Syst Rev. (2020) 7:Cd013600. doi: 10.1002/14651858.CD013600.pub2

16. Chai KL, Valk SJ, Piechotta V, Kimber C, Monsef I, Doree C, et al. Convalescent plasma or hyperimmune immunoglobulin for people with COVID-19: a living systematic review. Cochrane Datab Syst Rev. (2020) 10:Cd013600. doi: 10.1002/14651858.CD013600.pub3

17. Moher D, Liberati A, Tetzlaff J, Altman DG, Group P. Preferred reporting items for systematic reviews and meta-analyses: the PRISMA statement. Int J Surg. (2010) 8:336-41. doi: 10.1016/j.ijsu.2010.02.007

18. Moher D, Liberati A, Tetzlaff J, Altman DG, Prisma G. Preferred reporting items for systematic reviews and meta-analyses: the PRISMA statement. PLoS med. (2009) 6:e1000097. doi: 10.1371/journal.pmed.1000097

19. Higgins JPT, Altman DG, Gøtzsche PC, Jüni P, Moher D, Oxman AD, et al. The cochrane collaboration's tool for assessing risk of bias in randomised trials. BMJ. (2011) 343:d5928. doi: 10.1136/bmj.d5928

20. Balk EM, Bonis PA, Moskowitz H, Schmid CH, Ioannidis JP, Wang C, et al. Correlation of quality measures with estimates of treatment effect in meta-analyses of randomized controlled trials. JAMA. (2002) 287:2973-82. doi: 10.1001/jama.287.22.2973 
21. National Heart L, and Blood Institute. Quality Assessment Tool for Case Series Studies. (2021). Available online at: https://www.nhlbi.nih.gov/health-topics/ study-quality-assessment-tools (accessed January 31, 2021).

22. Wells GA SB, O'Connell D, Peterson J, Welch V, Losos M, Tugwell P. The Newcastle-Ottawa Scale (NOS) for Assessing the Quality of Nonrandomised Studies in Meta-Analyses. (2009). Available online at: http://www.ohri.ca/ programs/clinical_epidemiology/oxford.asp (accessed January 31, 2021).

23. The Cochrane Collaboration. Review Manager (RevMan) [Computer program]. Version 5.4 for Windows. Oxford: The Cochrane Collaboration. (2020). Available online at: https://training.cochrane.org/online-learning/ core-software-cochrane-reviews/revman/revman-5-download (accessed January 31, 2021).

24. Borenstein M, Hedges L, Higgins J, Rothstein H. Comprehensive MetaAnalysis Version 3. Englewood, NJ: Biostat. (2013).

25. Deeks JJ, Higgins JPT, Altman DG, Cochrane Statistical Methods G. Analysing data and undertaking meta-analyses. In: Higgins J, Thomas J, Chandler J, editors. Cochrane Handbook for Systematic Reviews of Interventions version 6.0. Chichester: John Wiley \& Sons (2019). p. 241-84. doi: 10.1002/9781119536604.ch10

26. Higgins JPT, Thomas J, Chandler J, Cumpston M, Li T, Page MJ, et al. Cochrane Handbook for Systematic Reviews of Interventions. Hoboken, NJ: John Wiley \& Sons (2019).

27. Abolghasemi H, Eshghi P, Cheraghali AM, Imani Fooladi AA, Bolouki Moghaddam F, Imanizadeh S, et al. Clinical efficacy of convalescent plasma for treatment of COVID-19 infections: Results of a multicenter clinical study. Transfus Apher Sci. (2020) 59:102875. doi: 10.1016/j.transci.2020.102875

28. Agarwal A, Mukherjee A, Kumar G, Chatterjee P, Bhatnagar T, Malhotra P. Convalescent plasma in the management of moderate covid-19 in adults in India: open label phase II multicentre randomised controlled trial (PLACID Trial). BMJ. (2020) 371:m3939. doi: 10.1136/bmj.m3939

29. Ahn JY, Sohn Y, Lee SH, Cho Y, Hyun JH, Baek YJ, et al. Use of convalescent plasma therapy in two COVID-19 patients with acute respiratory distress syndrome in Korea. J Korean Med Sci. (2020) 35:e149. doi: $10.3346 / \mathrm{jkms}$.2020.35.e149

30. Donato M, Park S, Baker M, Korngold R, Morawski A, Geng X, et al. Clinical and laboratory evaluation of patients with SARS-CoV-2 pneumonia treated with high-titer convalescent plasma: a prospective study. medRxiv [Preprint]. (2020). doi: 10.1101/2020.07.20.20156398

31. Gharbharan A, Jordans CCE, GeurtsvanKessel C, den Hollander JG, Karim F, Mollema FPN, et al. Convalescent plasma for COVID-19. A randomized clinical trial. medRxiv [Preprint]. (2020). doi: 10.1101/2020.07.01.20 139857

32. Hartman W, Hess AS, Connor JP. Hospitalized COVID-19 patients treated with convalescent plasma in a mid-size city in the Midwest. Translat Med Commun. (2020) 5:17. doi: 10.1186/s41231-020-00068-9

33. Hegerova L, Gooley TA, Sweerus KA, Maree C, Bailey N, Bailey M, et al. Use of convalescent plasma in hospitalized patients with COVID-19: case series. Blood. (2020) 136:759-62. doi: 10.1182/blood.2020006964

34. Li L, Zhang W, Hu Y, Tong X, Zheng S, Yang J, et al. Effect of convalescent plasma therapy on time to clinical improvement in patients with severe and life-threatening COVID-19: a randomized clinical trial. JAMA. (2020) 324:460-70. doi: 10.1001/jama.2020.10044

35. Liu STH, Lin HM, Baine I, Wajnberg A, Gumprecht JP, Rahman $\mathrm{F}$, et al. Convalescent plasma treatment of severe COVID-19: a propensity score-matched control study. Nat Med. (2020) 26:1708-13. doi: 10.1038/s41591-020-1088-9

36. Maor Y, Cohen D, Paran N, Israely T, Ezra V, Axelrod O, et al. Compassionate use of convalescent plasma for treatment of moderate and severe pneumonia in COVID-19 patients and association with $\operatorname{IgG}$ antibody levels in donated plasma. EClinicalMedicine. (2020) 26:100525. doi: 10.1016/j.eclinm.2020.100525

37. Martinez-Resendez MF, Castilleja-Leal F, Torres-Quintanilla A, RojasMartinez A, Garcia-Rivas G, Ortiz-Lopez R, et al. Initial experience in Mexico with convalescent plasma in COVID-19 patients with severe respiratory failure, a retrospective case series. medRxiv [Preprint]. (2020). doi: 10.1101/2020.07.14.20144469

38. Olivares-Gazca JC, Priesca-Marin JM, Ojeda-Laguna M, Garces-Eisele J, Soto-Olvera S, Palacios-Alonso A, et al. Infusion of convalescent plasma is associated with clinical improvement in critically ill patients with Covid19: a pilot study. Rev Invest Clin. (2020) 72:159-64. doi: 10.24875/RIC. 20000237

39. Pappa V, Politou M, Papageorgiou SG, Antoniadou A, Kotanidou A, Bouchla A, et al. A prospective phase II study on the use of convalescent plasma monotherapy for the treatment of severe Covid-19 disease: a preliminary report. Preprint. (2020). doi: 10.21203/rs.3.rs-64465/v1

40. Pei S, Yuan X, Zhimin Zhang Z, Run Yao R, Xie Y, Minxue Shen M, et al. Convalescent plasma to treat COVID-19: Chinese strategy and experiences. medRxiv [Preprint]. (2020). doi: 10.1101/2020.04.07.20056440

41. Perotti C, Baldanti F, Bruno R, Del Fante C, Seminari E, Casari S, et al. Mortality reduction in 46 severe Covid-19 patients treated with hyperimmune plasma. A proof of concept single arm multicenter trial. Haematologica. (2020) 105:2834-40. doi: 10.1101/2020.05.26.20113373

42. Rasheed AM, Ftak DF, Hashim HA, Maulood MF, Kabah KK, Almusawi YA, et al. The therapeutic effectiveness of Convalescent plasma therapy on treating COVID-19 patients residing in respiratory care units in hospitals in Baghdad, Iraq. medRxiv [Preprint]. (2020). doi: 10.1101/2020.06.24.20121905

43. Salazar E, Christensen PA, Graviss EA, Nguyen DT, Castillo B, Chen J, et al. Treatment of coronavirus disease 2019 patients with convalescent plasma reveals a signal of significantly decreased mortality. Am J Pathol. (2020) 190:2290-303. doi: 10.1016/j.ajpath.2020.08.001

44. Shen C, Wang Z, Zhao F, Yang Y, Li J, Yuan J, et al. Treatment of 5 critically ill patients with COVID-19 with convalescent plasma. JAMA. (2020) 323:1582-9. doi: 10.1001/jama.2020.4783

45. Tan L, Kang X, Zhang B, Zheng S, Liu B, Yu T, et al. A special case of COVID19 with long duration of viral shedding for 49 days. medRxiv [Preprint]. (2020). doi: 10.1101/2020.03.22.20040071

46. Wang M, Yang X, Yang F, Zhu X, Sun Z, Bao P, et al. Convalescent plasma therapy in critically ill coronavirus disease 2019 patients with persistently positive nucleic acid test, case series report. Medicine. (2020) 99:e21596. doi: 10.1097/MD.0000000000021596

47. Xia X, Li K, Wu L, Wang Z, Zhu M, Huang B, et al. Improved clinical symptoms and mortality among patients with severe or critical COVID-19 after convalescent plasma transfusion. Blood. (2020) 136:755-9. doi: 10.1182/blood.2020007079

48. Ye M, Fu D, Ren Y, Wang F, Wang D, Zhang F, et al. Treatment with convalescent plasma for COVID-19 patients in Wuhan, China. J Med Virol. (2020) 92:1890-901. doi: 10.1002/jmv.25882

49. Zeng QL, Yu ZJ, Gou JJ, Li GM, Ma SH, Zhang GF, et al. Effect of convalescent plasma therapy on viral shedding and survival in patients with coronavirus disease 2019. J Infect Dis. (2020) 222:38-43. doi: 10.1093/infdis/jiaa228

50. Zhang B, Liu S, Tan T, Huang W, Dong Y, Chen L, et al. Treatment with convalescent plasma for critically ill patients with severe acute respiratory syndrome coronavirus 2 infection. Chest. (2020) 158:e9-13. doi: 10.1016/j.chest.2020.03.039

51. Karekadavath N, Pullichola SB, Afsal EM, Prabhu ASP, Abdulla MC, Ebrahim SH. Convalescent plasma therapy in first four critically ill COVID-19 patients in Kerala, India. Dr Sulaiman Al Habib Med J. (2020) 2:87-91. doi: 10.2991/dsahmj.k.200908.001

52. Erkurt MA, Sarici A, Berber I, Kuku I, Kaya E, Özgül M. Lifesaving effect of convalescent plasma treatment in covid-19 disease: clinical trial from eastern Anatolia. Transfus Apher Sci. (2020) 59:102867. doi: 10.1016/j.transci.2020.102867

53. Omrani AS, Zaqout A, Baiou A, Daghfal J, Elkum N, Alattar RA, et al. Convalescent plasma for the treatment of patients with severe coronavirus disease 2019: a preliminary report. J Med Virol. (2020) 93:1678-86. doi: 10.1002/jmv.26537

54. Altuntas F, Ata N, Yigenoglu TN, Basci S, Dal MS, Korkmaz S, et al. Convalescent plasma therapy in patients with COVID-19. Transfus Apher Sci. (2020) 102955. doi: 10.1016/j.transci.2020.102955

55. Rogers R, Shehadeh F, Mylona EK, Rich J, Neill M, Touzard-Romo F, et al. Convalescent plasma for patients with severe COVID-19: a matched cohort study. Clin Infect Dis. (2020) ciaa1548. doi: 10.1093/cid/ciaa1548

56. Simonovich VA, Burgos Pratx LD, Scibona P, Beruto MV, Vallone MG, Vázquez C, et al. A randomized trial of convalescent plasma in Covid-19 severe pneumonia. N Engl J Med. (2020) 384:619-29. doi: 10.1056/NEJMoa2031304 
57. Yoon Ha, Bartash R, Gendlina I, Rivera J, Nakouzi A, Bortz RH, et al. Treatment of severe COVID-19 with convalescent plasma in the bronx, NYC. medRxiv [Preprint]. (2020). doi: 10.1101/2020.12.02.202 42909

58. Libster R, Marc GP, Wappner D, Coviello S, Bianchi A, Braem V, et al. Prevention of severe COVID-19 in the elderly by early hightiter plasma. medRxiv [Preprint]. (2020). doi: 10.1101/2020.11.20.202 34013

59. Bajpai M, Kumar S, Maheshwari A, Chhabra K, kale P, Gupta A, et al. Efficacy of convalescent plasma therapy compared to fresh frozen plasma in severely ill COVID-19 patients: a pilot randomized controlled trial. medRxiv [Preprint]. (2020). doi: 10.1101/2020.10.25.20219337

60. AlQahtani M, Abdulrahman A, Almadani A, Alali SY, Al Zamrooni AM, Hejab AH, et al. Randomized controlled trial of convalescent plasma therapy against standard therapy in patients with severe COVID-19 disease. medRxiv [Preprint]. (2020). doi: 10.1101/2020.11.02.20224303

61. Ray Y, Paul SR, Bandopadhyay P, D’Rozario R, Sarif J, Lahiri A, et al. Clinical and immunological benefits of convalescent plasma therapy in severe COVID-19: insights from a single center open label randomised control trial. medRxiv [Preprint]. (2020). doi: 10.1101/2020.11.25.202 37883

62. Avendano-Sola C, Ramos-Martinez A, Munez-Rubio E, Ruiz-Antoran B, Malo de Molina R, Torres F, et al. Convalescent plasma for COVID19: a multicenter, randomized clinical trial. medRxiv [Preprint]. (2020). doi: 10.1101/2020.08.26.20182444

63. Luke TC, Kilbane EM, Jackson JL, Hoffman SL. Meta-analysis: convalescent blood products for Spanish influenza pneumonia: a future H5N1 treatment? Ann Intern Med. (2006) 145:599-609. doi: 10.7326/0003-4819-145-8-200610170-00139

64. Zhou B, Zhong N, Guan Y. Treatment with convalescent plasma for influenza A (H5N1) infection. N Engl J Med. (2007) 357:1450-1. doi: 10.1056/NEJMc070359

65. World Health Organization. Use of Convalescent Whole Blood or Plasma Collected from Patients Recovered from Ebola Virus Disease for Transfusion, as an Empirical Treatment during Outbreaks Interim Guidance for National Health Authorities and Blood Transfusion Services Use of Convalescent Whole Blood or Plasma Collected from Patients Recovered from Ebola Virus Disease for Transfusion, as an Empirical Treatment during Outbreaks. Geneva (2014).

66. Hung IF, To KK, Lee CK, Lee KL, Chan K, Yan WW, et al. Convalescent plasma treatment reduced mortality in patients with severe pandemic influenza A (H1N1) 2009 virus infection. Clin Infect Dis. (2011) 52:447-56. doi: $10.1093 / \mathrm{cid} / \mathrm{ciq} 106$
67. Cheng Y, Wong R, Soo YO, Wong WS, Lee CK, $\mathrm{Ng} \mathrm{MH}$, et al. Use of convalescent plasma therapy in SARS patients in Hong Kong. Eur J Clin Microbiol Infect Dis. (2005) 24:44-6. doi: 10.1007/s10096-004-1271-9

68. Robbiani DF, Gaebler C, Muecksch F, Lorenzi JCC, Wang Z, Cho A, et al. Convergent antibody responses to SARS-CoV-2 in convalescent individuals. Nature. (2020) 584:437-42. doi: 10.1038/s41586-020-2456-9

69. Klein SL, Pekosz A, Park HS, Ursin RL, Shapiro JR, Benner SE, et al. Sex, age, and hospitalization drive antibody responses in a COVID-19 convalescent plasma donor population. J Clin Invest. (2020) 130:6141-50. doi: 10.1172/JCI142004

70. Busch MP, Bloch EM, Kleinman S. Prevention of transfusion-transmitted infections. Blood. (2019) 133:1854-64. doi: 10.1182/blood-2018-11-833996

71. Hendrickson JE, Hillyer CD. Noninfectious serious hazards of transfusion. Anesth Analg. (2009) 108:759-69. doi: 10.1213/ane.0b013e3181 930a6e

72. The Standards Program Committee (SPC) and the Blood Banks and Transfusion Services Standards Committee (BB/TS SC). Standards for Blood Banks and Transfusion Services. Bethesda, MD: American Association of Blood Banks (2018).

73. Bloch EM, Shoham S, Casadevall A, Sachais BS, Shaz B, Winters JL, et al. Deployment of convalescent plasma for the prevention and treatment of COVID-19. J Clin Invest. (2020) 130:2757-65. doi: 10.1172/JCI138745

74. Garg AX, Hackam D, Tonelli M. Systematic review and meta-analysis: when one study is just not enough. Clin J Am Soc Nephrol. (2008) 3:253-60. doi: 10.2215/CJN.01430307

75. Walkey AJ, Kumar VK, Harhay MO, Bolesta S, Bansal V, Gajic $\mathrm{O}$, et al. The viral infection and respiratory illness universal study (VIRUS): an international registry of coronavirus 2019-related critical illness. Crit Care Explor. (2020) 2:e0113. doi: 10.1097/CCE.0000000000 000113

Conflict of Interest: The authors declare that the research was conducted in the absence of any commercial or financial relationships that could be construed as a potential conflict of interest.

Copyright (C) 2021 Bansal, Mahapure, Mehra, Bhurwal, Tekin, Singh, Gupta, Rathore, Khan, Deshpande, Gulati, Armaly, Sheraton and Kashyap. This is an openaccess article distributed under the terms of the Creative Commons Attribution License (CC BY). The use, distribution or reproduction in other forums is permitted, provided the original author(s) and the copyright owner(s) are credited and that the original publication in this journal is cited, in accordance with accepted academic practice. No use, distribution or reproduction is permitted which does not comply with these terms. 